\title{
Multidimensional gene regulatory landscape of a bacterial pathogen in plants
}

\author{
Tatsuya Nobori ${ }^{1,4,8}$, Yiming Wang ${ }^{1,5,8}$, Jingni Wu ${ }^{1,6}$, Sara Christina Stolze $\mathbb{\circledR}^{2}$, Yayoi Tsuda ${ }^{1,3}$, \\ Iris Finkemeier ${ }^{2,7}$, Hirofumi Nakagami ${ }^{2}$ and Kenichi Tsuda $1,3 \bowtie$
}

\begin{abstract}
Understanding the gene regulation of plant pathogens is crucial for pest control and thus global food security. An integrated understanding of bacterial gene regulation in the host is dependent on multi-omic datasets, but these are largely lacking. Here, we simultaneously characterized the transcriptome and proteome of a bacterial pathogen in plants. We found a number of bacterial processes affected by plant immunity at the transcriptome and proteome levels. For instance, salicylic acid-mediated plant immunity suppressed the accumulation of proteins comprising the tip component of the bacterial type III secretion system. Interestingly, there were instances of concordant and discordant regulation of bacterial messenger RNAs and proteins. Gene co-expression analysis uncovered previously unknown gene regulatory modules underlying virulence. This study provides molecular insights into the multiple layers of gene regulation that contribute to bacterial growth in planta, and elucidates the role of plant immunity in affecting pathogen responses.
\end{abstract}

T he growth of bacterial pathogens in plants and disease development are determined by genetically encoded bacterial virulence and plant immune systems ${ }^{1}$. Despite the wealth of available knowledge concerning these systems in isolation, interactions between the two-especially how plant immunity affects bacterial function-are poorly understood ${ }^{2}$. Previously, it was shown that in planta transcriptomics of the bacterial pathogen Pseudomonas syringae pv. tomato DC3000 (Pto) can be used to identify bacterial messenger RNAs whose expression is influenced by plant immune activation ${ }^{3}$. The study investigated the roles of two major forms of plant immunity, pattern-triggered immunity (PTI) and effector-triggered immunity (ETI), in affecting the transcriptomes of Pto in Arabidopsis thaliana leaves at an early stage of infection. ETI was triggered using Pto strains expressing effectors such as AvrRpt2 (ref. ${ }^{4,5}$ ). Activation of PTI or ETI dramatically changed the transcriptomes of Pto, and the degree of transcriptome changes correlated well with bacterial growth in plants. Because the salicylic acid (SA) pathways are important components of $\mathrm{ETI}^{6}$, the impact of ETI on bacterial transcriptomes was strongly compromised in single and double mutants of SID2 and PAD4, key components of the SA pathways, as well as the AvrRpt2 receptor mutant rps2 rpm1. Intriguingly, a bacterial iron-acquisition pathway was commonly suppressed by PTI and ETI. In planta transcriptome analysis has also been conducted in fungal pathogens. One study profiled co-transcriptomes of A. thaliana and a variety of Botrytis cinerea isolates to reveal gene co-expression networks of the pathogens that underlie virulence mechanisms ${ }^{7}$. Another study analysed transcriptomes of the fungal pathogen Colletotrichum at multiple stages of infection in A. thaliana and maize, revealing transcriptome signatures associated with the lifestyle transition of the fungal pathogens in planta ${ }^{8}$, highlighting that analysis of pathogen responses at different infection stages can also be informative.
Although transcriptome analysis is a useful and widely used approach for elucidation of cellular function, it has been well established that mRNA expression does not always reflect protein expression and thus it becomes clear that a better understanding of cellular behaviour requires direct interrogation of protein expression ${ }^{9,10}$. A previous study showed that proteome analysis of leaf commensal bacteria can reveal metabolic changes in bacteria residing on the leaf surface ${ }^{11}$. However, the capacity of proteomics to describe plant-associated bacteria remains limited ${ }^{12}$. For instance, analysis of bacterial responses in the intercellular space (apoplast) of leaves, which is an important niche for various commensal and pathogenic bacteria, poses a major challenge because the large preponderance of plant material relative to bacterial material confounds analysis. To date, there is no proteome study of bacterial pathogens in the leaf apoplast and, thus, we lack comprehensive knowledge of bacterial proteins that are affected by host plants during infection. Moreover, due to the lack of comparative analyses spanning different modalities of bacterial responses in planta, little is known about the flow of bacterial genetic information (for example, from mRNA expression to protein expression) that is important for virulence and how this is affected by plant immunity.

Here, we simultaneously profiled bacterial transcriptomes and proteomes in planta and identified bacterial processes influenced by plant immunity at the mRNA and protein levels at early and late stages of infection. Comparative analysis of transcriptomes and proteomes revealed that changes in bacterial mRNA and protein expression are correlated in general. However, there are functions that are selectively changed at the mRNA or protein level. Furthermore, gene regulatory network analysis of bacteria showed previously unknown gene regulatory modules that mediate bacterial virulence in planta. Together, this study reveals the multi-layered regulatory mechanisms that underlie interactions between plants and bacterial pathogens.

'Department of Plant Microbe Interactions, Max Planck Institute for Plant Breeding Research, Cologne, Germany. ${ }^{2}$ Protein Mass Spectrometry Group, Max Planck Institute for Plant Breeding Research, Cologne, Germany. ${ }^{3}$ State Key Laboratory of Agricultural Microbiology, College of Plant Science and Technology, Huazhong Agricultural University, Wuhan, China. ${ }^{4}$ Present address: Salk Institute for Biological Studies, La Jolla, CA, USA. ${ }^{5}$ Present address: College of Plant Protection, Nanjing Agriculture University, Nanjing, China. ${ }^{6}$ Present address: National Key Laboratory of Plant Molecular Genetics, Shanghai Institute of Plant Physiology and Ecology, Chinese Academy of Sciences, Shanghai, China. ${ }^{7}$ Present address: Institute of Plant Biology and Biotechnology, University of Muenster, Münster, Germany. ${ }^{8}$ These authors contributed equally: Tatsuya Nobori, Yiming Wang. ${ }^{\circledR e}$-mail: tsuda@mail.hzau.edu.cn 


\section{Results}

In planta transcriptome and proteome profiling of $P$. syringae. We profiled the transcriptome and proteome of the bacterial pathogen Pto in A. thaliana leaves using RNA-sequencing (RNA-seq) and liquid chromatography-mass spectrometry (LC-MS), respectively. Bacterial information was enriched by isolating bacterial cells from infected plant leaves using a previously established method (Fig. 1a) ${ }^{3}$. Briefly, infected leaves were crushed and incubated in a buffer that stops bacterial metabolism and protects bacterial RNA from degradation. After separation of bacterial and plant cells by centrifugation, RNA and protein were extracted from isolated bacteria and subjected to RNA-seq and LC-MS analysis, respectively (Fig. 1a). Both the transcriptomes and proteomes of Pto were profiled under 15 conditions, including 2 in vitro and 13 in planta (Fig. 1b). Two bacterial strains, the virulent Pto and the ETI-triggering Pto AvrRpt2, were used. For genetic dissection of the roles of SA pathways and ETI, we used the single and double mutants of SID2 and PAD4, and rps2 rpm1, which lacks the AvrRpt2 receptor RPS2. In total, 70 and 45 samples were analysed for transcriptome and proteome, respectively (Extended Data Fig. 1a,b and Supplementary Data 13). Note that this is not a time-course study, because the doses of starting bacterial inocula are different for the two time points $\left(\mathrm{OD}_{600}=0.5\right.$ for $6 \mathrm{~h}$ post infection (hpi) and $\mathrm{OD}_{600}=0.005$ for $48 \mathrm{hpi}$ ). A higher dose of bacteria was used for $6 \mathrm{hpi}$, because efficient bacterial isolation requires a large population of bacteria in leaves; and a lower dose was used for $48 \mathrm{hpi}$ because inoculation of the higher dose of Pto could kill plants at this time point. Pto AvrRpt2 was not used for the sampling at $48 \mathrm{hpi}$, because this strain caused tissue collapse in the leaves even under this condition. In the proteome analysis, we could detect up to approximately 2,000 proteins in planta (Fig. 1c). Regardless of conditions, detected mRNAs/proteins showed protein subcellular distribution similar to total proteins encoded in the Pto genome (Fig. 1c), suggesting that no obvious bias was introduced into $\mathrm{mRNA}$ /protein detection during bacterial enrichment processes. Pto showed distinct responses under different conditions at both transcriptome (Fig. 1d,e) and proteome (Fig. 1f,g) levels. In vitro bacterial transcriptome/proteome patterns were more distinct from in planta expression patterns at 6 hpi compared with 48 hpi (Fig. 1d-g), suggesting that dynamic transcriptional reprogramming at an early stage of infection is crucial for Pto in adapting to the plant apoplastic environment and becoming virulent. Although different doses of starting inocula between different time points may explain the difference, this notion is supported by our previous observation that bacterial transcriptome patterns at this early stage of infection can predict bacterial growth in plants at a later stage ${ }^{3}$.

Dynamic regulation of bacterial function across different conditions. We analysed mRNAs and proteins whose expression was significantly ( $\mid \log _{2}[$ fold change $(\mathrm{FC})] \mid>1$; FDR $<0.01$; two-tailed Student's $t$-test followed by Storey's $q$-value) changed between the in vitro King's B medium (KB) and in planta (Col-0) conditions at 6 or 48 hpi. Gene ontology (GO) enrichment analysis showed that mRNAs, as well as proteins related to 'pathogenesis', 'translation' and 'cell wall organization or biogenesis', were induced at both 6 and $48 \mathrm{hpi}$ (Clusters I and II in Fig. 2a and Cluster I in Fig. 2b). Interestingly, in the proteome data, transfer RNA synthases and ribosomal proteins, both of which are related to protein translation, showed the opposite expression patterns-that is, tRNA synthases were suppressed at $48 \mathrm{hpi}$ while ribosomal proteins were induced (Clusters I and III in Fig. 2b). Whether bacterial translation efficiency is reduced or enhanced at 48 hpi compared with the other conditions remains to be elucidated. A substantial number of Pto mRNAs (324) and proteins (196) were differentially expressed among different host genotypes at 48 hpi (Fig. 2c,d), whereas host genotype effects were small at $6 \mathrm{hpi}$ (only $15 \mathrm{mRNAs}$ and one protein were differentially expressed, respectively). Pto AvrRpt 2 was strongly affected by the plant SA pathway at $6 \mathrm{hpi}$ at the transcriptome level ${ }^{3}$, but not at the proteome level (only one protein was differentially expressed). This might be because transcriptional changes were not yet reflected in protein accumulation at $6 \mathrm{hpi}$. Thus, we focused on analysis of host genotype effects on protein expression in Pto at $48 \mathrm{hpi}$. There were 196 proteins whose expression was significantly $\left(\left|\log _{2}[\mathrm{FC}]\right|>1\right.$; FDR $<0.01$; two-tailed Student's $t$-test followed by Storey's $q$-value) affected in at least one of the SA mutants (Fig. 2d). GO enrichment analysis revealed that pathogenesis-related proteins (interaction with host) were highly expressed in the SA mutants (Cluster II in Fig. 2d), implying that the SA immune pathways suppress expression of pathogenesis-related proteins. On the other hand, expression of translation-related proteins (ribosomal proteins) was reduced in the SA mutants (Cluster I in Fig. 2d). Taken together, the SA pathway affects expression of bacterial proteins related to bacterial virulence and basic metabolism in planta.

As shown in Fig. 2, bacterial functions differentially expressed under different conditions could be studied by GO enrichment analysis following differential expression analysis. However, this analysis is highly dependent on the thresholds applied for selection of differentially expressed mRNAs or proteins, and thus some important information can be lost before GO enrichment analysis. Moreover, it is difficult to compare the global expression pattern of GO terms across many conditions using this approach. To gain insights into biological functions that are differentially regulated in various conditions, we grouped Pto genes into GO terms and calculated standardized GO expression scores; GO terms expressed in a highly condition-dependent manner were then selected (Fig. 3a,b; see Methods). The GO term pathogenesis was one of the most dynamically regulated processes at both the transcriptome and proteome levels (Fig. 3a,b). These mRNAs and proteins were strongly induced in planta at $6 \mathrm{hpi}$; their expression remained high at $48 \mathrm{hpi}$, and a clear host genotype effect was observed at this time point-that is, expression was higher in the mutants of the SA pathway (sid2, pad4, pad4 sid2) compared with the wild-type Col-0 (Fig. 3c,d), implying that SA-mediated immunity suppresses pathogenesis-related factors at the transcript level at $48 \mathrm{hpi}$. A similar pattern was observed in mRNAs and proteins related to the bacterial toxin, coronatine (Extended Data Fig. 3a). We found that successful activation of ETI strongly induced mRNAs/proteins related to 'catalase activity' at 6 hpi (Extended Data Fig. 3b), which probably reflects bacterial responses to oxidative burst, a hallmark of ETI responses. Interestingly these mRNAs and proteins were even more highly expressed in virulent $P$ to at 48 hpi (Extended Data Fig. 3b), suggesting that Pto experiences oxidative stress at later infection stages. Taken together, our multi-omic Pto dataset uncovered dynamic regulation of various biological processes across different conditions at the mRNA and protein levels.

Previously, we showed that bacterial genes related to the iron-acquisition pathway ('iron-starvation' genes) are highly induced in susceptible plants and strongly suppressed by the activation of PTI and/or ETI at 6 hpi at the mRNA level ${ }^{3}$ (Fig. 3e). Expression of these genes was lower at $48 \mathrm{hpi}$ compared with $6 \mathrm{hpi}$ (Fig. 3e), but still higher than under in vitro conditions. Expression of iron-starvation genes is known to be regulated by the master regulator protein Fur, which typically functions as a transcriptional repressor when bound by $\mathrm{Fe}(\mathrm{II})^{13}$. Interestingly, the accumulation of Fur protein was negatively correlated with the expression of iron-starvation genes in three distinct conditions (in vitro, in planta $6 \mathrm{hpi}$ and in planta 48 hpi; Fig. 3e,f). This implies a previously unknown mechanism of bacterial iron acquisition by which accumulation of the Fur protein might also contribute to regulation of iron-starvation genes.

Comparative analysis of bacterial transcriptomes and proteomes. To compare global expression patterns of genes and proteins, the transcriptome and proteome data of all 15 conditions were 
a

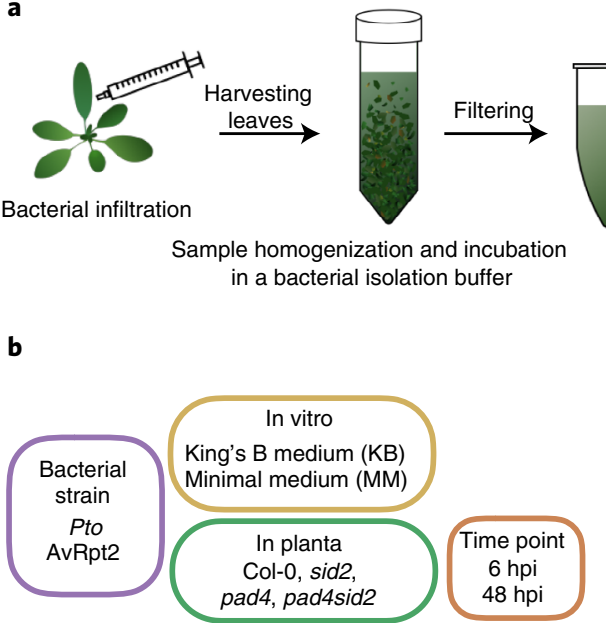

d
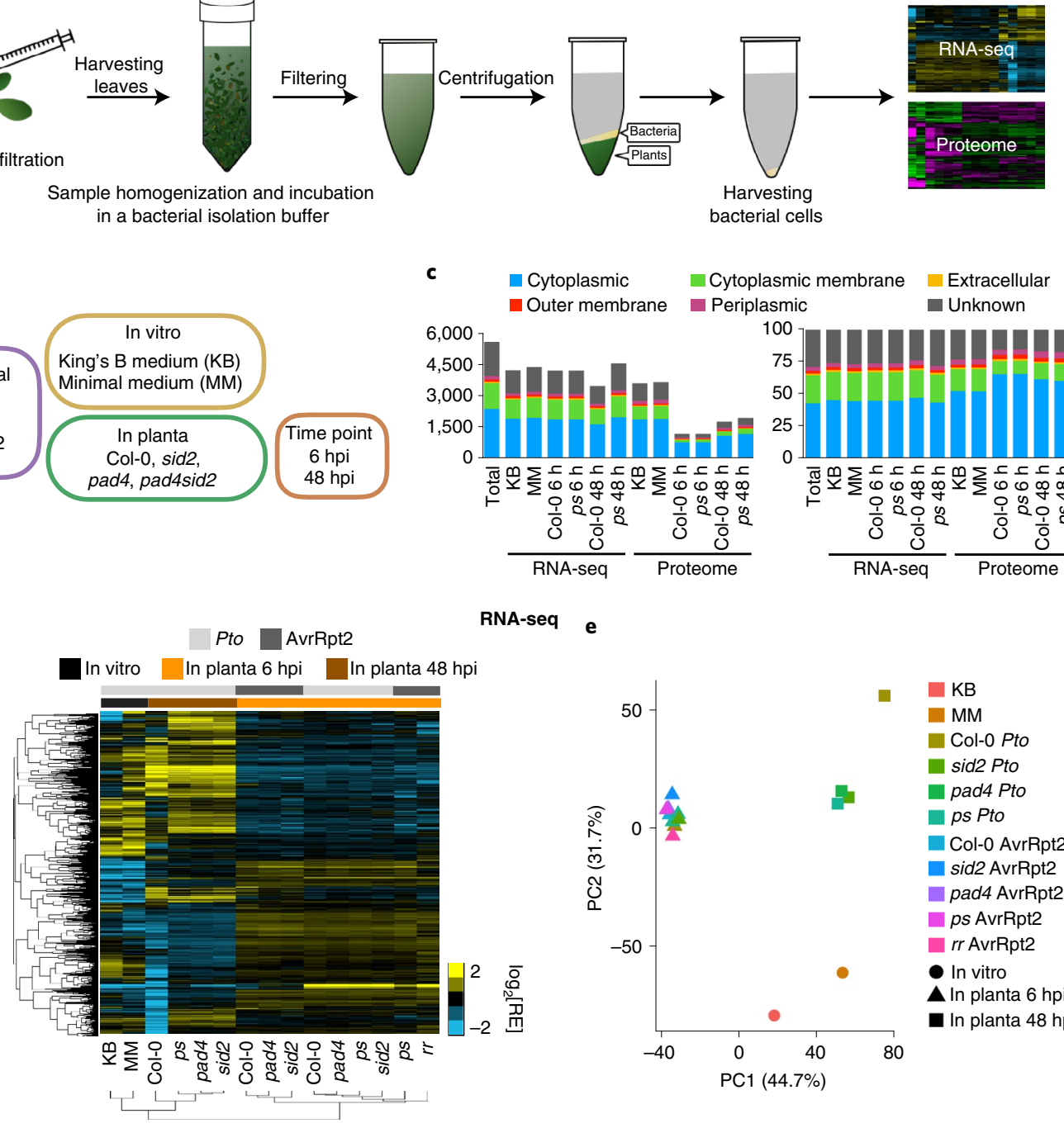
acterial cells

seq

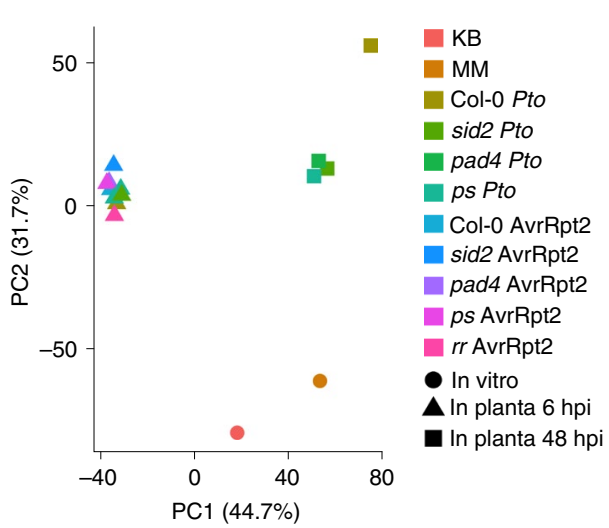

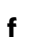

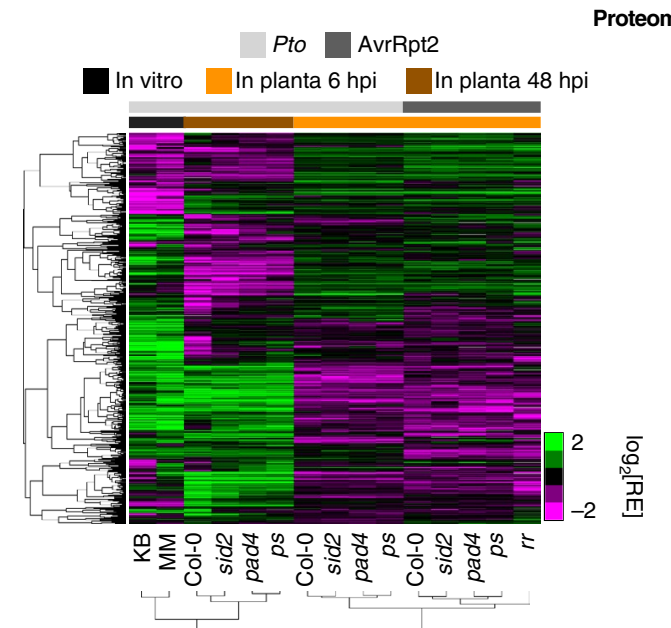

g

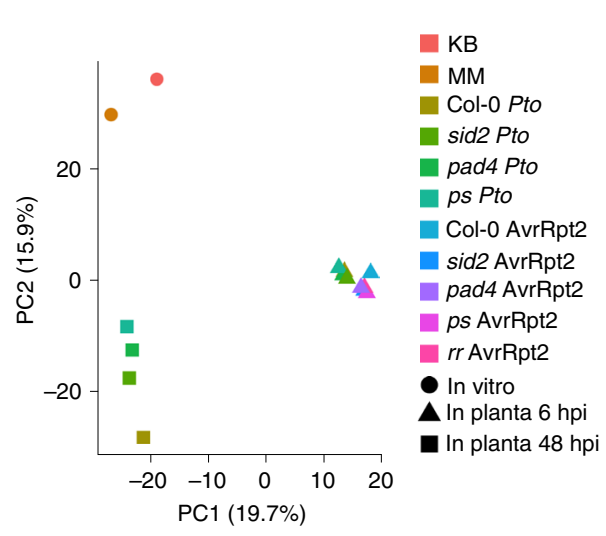

Fig. 1 | In planta transcriptomics and proteomics of Pto. a, Schematic workflow of in planta bacterial transcriptome and proteome analysis. b, Bacterial strains, plant genotypes and conditions used in this study. In total, 70 and 45 samples were analysed for transcriptome and proteome, respectively. See Extended Data Fig. 1 for an overview of the datasets. c, Number (left) and proportion (right) of mRNAs/proteins detected in the RNA-seq/proteome analysis, together with protein localization information. Total: all annotated genes of Pto. ps, pad4 sid2; rr, rpm1 rps2. d, Hierarchical clustering of relative expression (RE) of commonly detected Pto and Pto AvrRpt2 genes (total, 4,868) in RNA-seq analysis. See Supplementary Data 1 for gene expression data. e, Principle component analysis of Pto and Pto AvrRpt2 genes commonly detected in RNA-seq analysis. f, Hierarchical clustering of the relative expression of Pto and Pto AvrRpt2 proteins commonly detected in proteome analysis (937 proteins). See Supplementary Data 1 for the expression of proteins detected under at least one condition (2,018 proteins). In d,f, valid clusters of samples determined by $k$-mean clustering are shown in Supplementary Data 14 . Light and dark grey sidebars represent Pto and Pto AvrRpt2, respectively. Black, orange and brown sidebars represent in vitro (KB), in planta 6 hpi and in planta 48 hpi, respectively. $\mathbf{g}$, Principle component analysis of Pto and Pto AvrRpt2 proteins commonly detected in proteome analysis. For $\mathbf{d - g}$, see Supplementary Data 13 for sample sizes. 
a

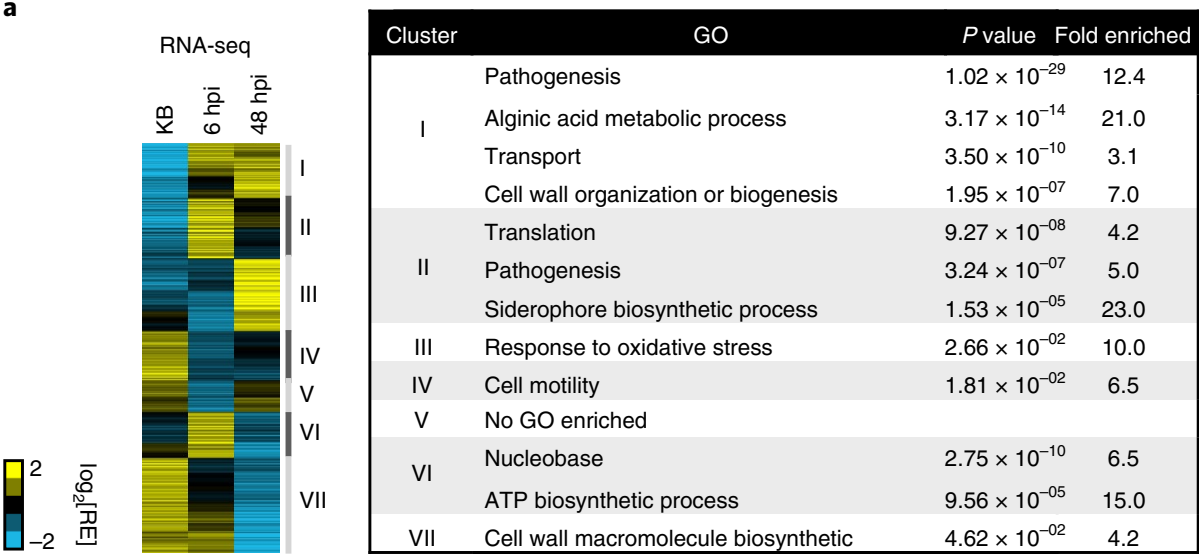

b

Proteome

\begin{tabular}{|c|c|c|c|}
\hline Cluster & GO & $P$ value $\mathrm{F}$ & Fold enriched \\
\hline \multirow{3}{*}{ I } & Translation (ribosomal proteins) & $2.59 \times 10^{-13}$ & 10.4 \\
\hline & Pathogenesis & $8.61 \times 10^{-11}$ & 11.2 \\
\hline & Cell wall organization or biogenesis & $1.76 \times 10^{-06}$ & 10.7 \\
\hline ॥ & Pathogenesis & $6.69 \times 10^{-03}$ & 8.1 \\
\hline \multirow{2}{*}{ III } & Nucleobase & $6.46 \times 10^{-09}$ & 8.8 \\
\hline & tRNA metabolic process & $1.12 \times 10^{-05}$ & 8.1 \\
\hline IV & Response to oxidative stress & $7.20 \times 10^{-03}$ & 22.0 \\
\hline V & Amine catabolic process & $3.33 \times 10^{-03}$ & 10.5 \\
\hline VI & Amine biosynthetic process & $2.47 \times 10^{-04}$ & 5.3 \\
\hline VII & Cell wall biogenesis & $6.05 \times 10^{-03}$ & 9.6 \\
\hline
\end{tabular}

c

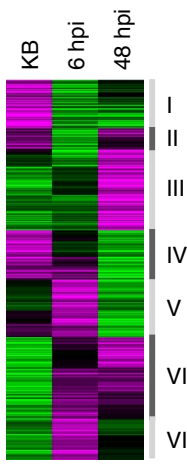

RNA-seq
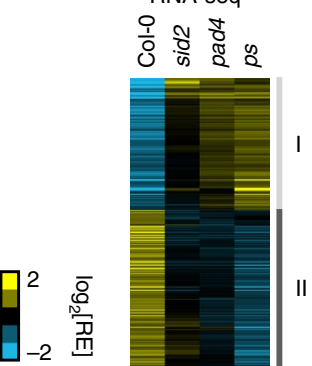

\section{Cluster}

GO

$P$ value Fold enriched

\begin{tabular}{|cll|}
\hline I & Pathogenesis $4.99 \times 10^{-30}$ & 19.4 \\
II $\quad$ No GO enriched & \\
\hline
\end{tabular}

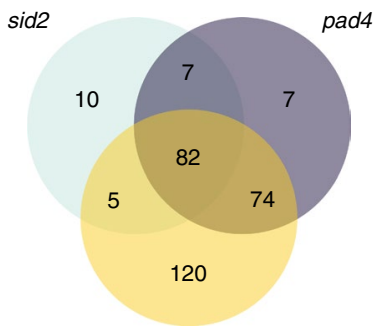

ps

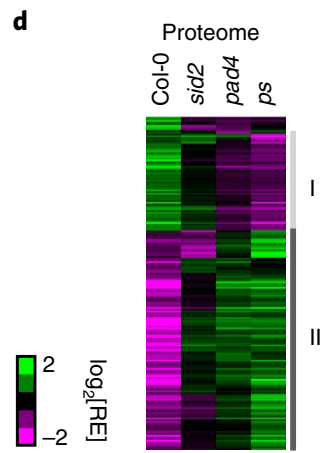

\begin{tabular}{|c|c|c|c|}
\hline Cluster & GO & $P$ value $\mathrm{F}$ & enriched \\
\hline 1 & Translation & $4.70 \times 10^{-08}$ & 6.5 \\
\hline \multirow{3}{*}{ II } & Pathogenesis (T3SS) & $6.84 \times 10^{-03}$ & 2.7 \\
\hline & Chemotaxis & $1.98 \times 10^{-04}$ & 2.5 \\
\hline & Organic acid metabolic & $2.37 \times 10^{-07}$ & 6.2 \\
\hline
\end{tabular}

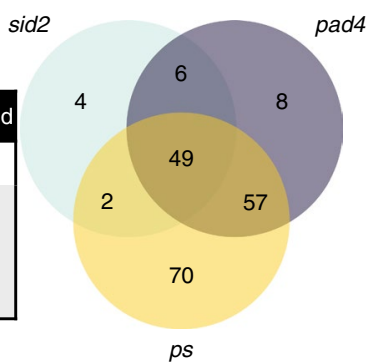

Fig. 2 | Distinct patterns of Pto transcriptomes and proteomes under various conditions. a,b, Hierarchical clustering of the relative expression (RE) of Pto mRNAs (a) and proteins (b) differentially expressed under at least one condition among in vitro (KB) and in planta conditions (Col-0) at 6 and 48 hpi $\left(\left|\log _{2}[F C]\right|>1 ; F D R<0.01\right.$; two-tailed Student's $t$-test followed by Storey's $q$-value). A list of GO terms enriched in the clusters is shown. $\mathbf{c}, \mathbf{d}$, Hierarchical clustering of the relative expression of Pto mRNAs (c) and proteins (d) differentially expressed in at least one plant SA mutant compared with the wild-type Col-0 at 48 hpi ( $\left|\log _{2}[F C]\right|>1 ; F D R<0.01$; two-tailed Student's $t$-test followed by Storey's $q$-value). A list of GO terms enriched in the clusters is shown. Venn diagrams showing Pto mRNAs/proteins differentially expressed in each plant mutant compared with Col-0. For mRNA/protein expression data and full GO lists, see Supplementary Data 2-6. ps, pad4 sid2. For a-d, see Supplementary Data 13 for sample sizes. 
a

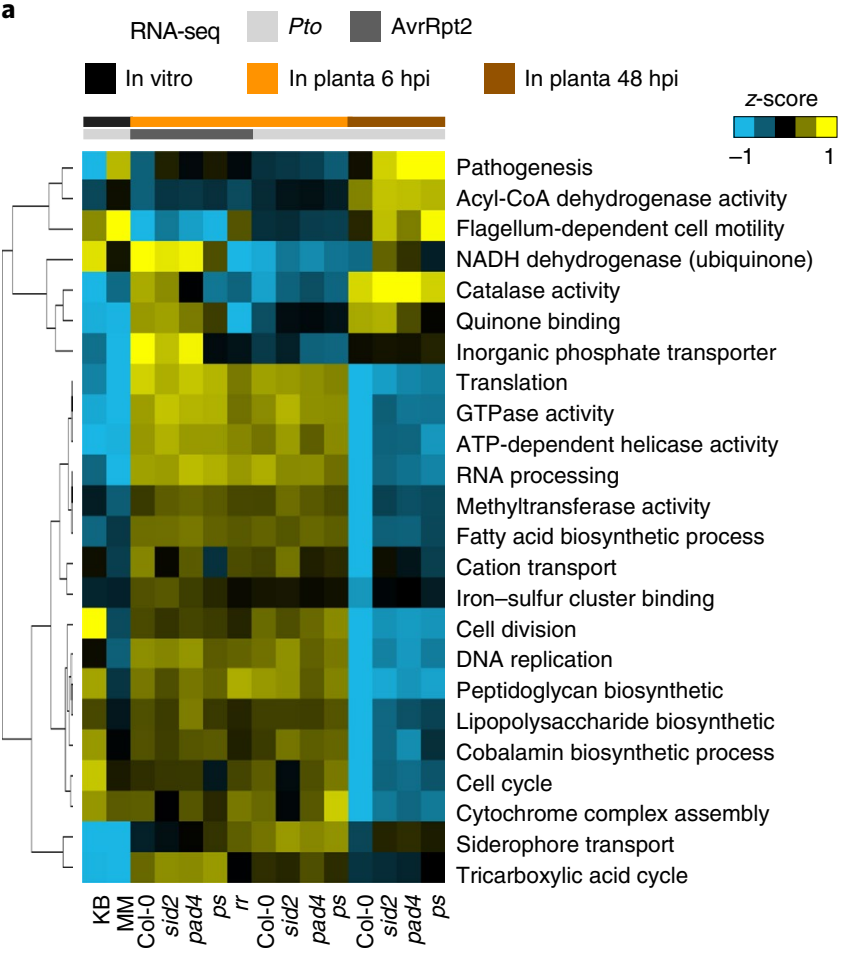

b

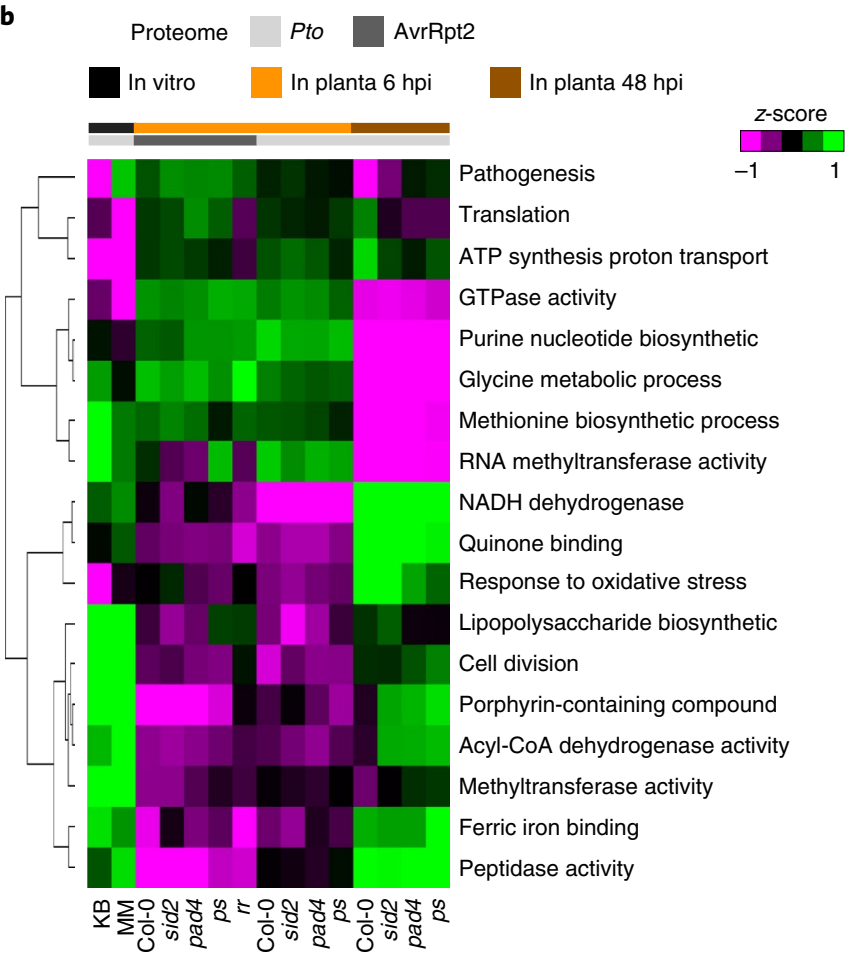

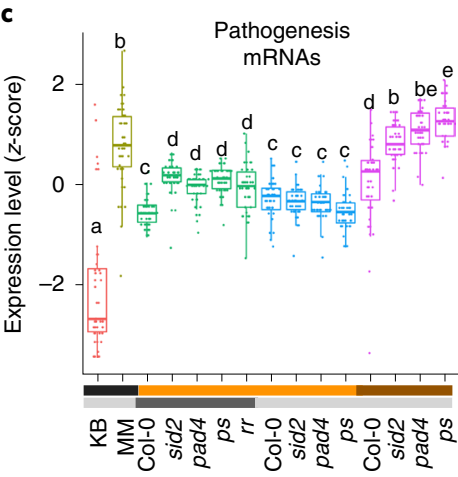
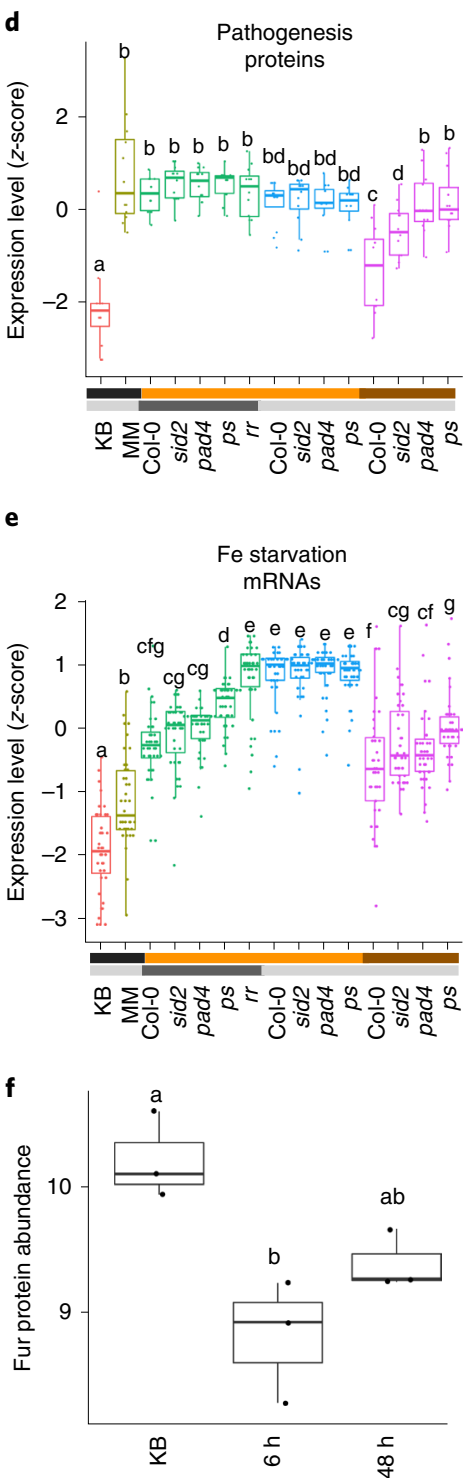

Fig. 3 Dynamic regulation of bacterial function across different conditions. a,b, Hierarchical clustering of selected GO terms in the transcriptome (a) and proteome (b) data. The transcriptome and proteome data were separately standardized using $\log _{2}[z$-scores], and mean $z$-scores of $m R N A s / p r o t e i n s$ involved in individual GO terms are shown. For the full GO expression data, see Supplementary Data 7. c,d, Box plots showing expression (z-score) of mRNAs (c) and proteins (d) related to pathogenesis. e, Box plots showing expression ( $z$-score) of mRNAs previously described as iron starvation genes ${ }^{43}$. In a-e, light and dark grey sidebars represent Pto and Pto AvrRpt2, respectively. Black, orange and brown sidebars represent in vitro (KB), in planta 6 hpi and in planta 48 hpi, respectively. ps, pad4 sid2; rr, rpm1 rps2. f, Expression of the Fur protein in Pto based on proteome data ( $\log _{2}$ [normalized iBAQ value]). For a-f, see Supplementary Data 13 for sample sizes. In c-f, results are shown as box plots with boxes displaying the 25th-75th percentiles, the centre line indicating the median and whiskers extending to the minimum and maximum values no further than $1.5 \times$ interquartile range. All individual data points are overlaid. Different letters indicate statistically significant differences (adjusted $P<0.01$, two-tailed Student's $t$-test, Benjamini-Hochberg method). 
standardized and combined, and hierarchical clustering was performed (Fig. 4a). Strikingly, transcriptome and proteome data were clustered together in three major conditions (in vitro and in planta $6 / 48 \mathrm{hpi}$ ) (Fig. 4a), indicating that the global patterns of bacterial gene expression and protein expression are similar both in vitro and in planta. Since many of the samples used for transcriptome and proteome data were prepared independently, the overall agreement between transcriptome and proteome data indicates the high accuracy of both sets of omics data.

We compared RNA-seq and proteome data under each condition. Under $\mathrm{KB}$ and minimal medium (MM) conditions, transcriptomes and proteomes were moderately correlated $\left(R^{2}=0.52\right.$ and 0.43 , respectively; Extended Data Fig. 4), which is consistent with previous studies in Escherichia coli $\left(R^{2}=0.42-0.57\right)^{14-16}$. A similar level of correlation was observed in planta, with slightly higher correlation at $6 \mathrm{hpi}\left(R^{2}=0.51-0.55\right)$ than at 48 hpi $\left(R^{2}=0.39-0.47\right.$; Extended Data Fig. 4). Thus, Pto mRNA and protein expression are moderately correlated both in vitro and in planta.

We further compared fold changes in RNA-seq and proteome data between Pto in vitro (KB) and each of the other conditions (Fig. 4b). In all conditions, expression changes in the transcriptome and proteome were moderately correlated $\left(R^{2}=0.52-0.63\right)$, suggesting that bacterial protein expression changes closely mirror those in mRNA levels during infection to both resistant and susceptible plants. Of 1,068 mRNAs/proteins detected in both RNA-seq and proteome analyses, $111(10.4 \%)$ were significantly $\left(\left|\log _{2}[\mathrm{FC}]\right|>1\right.$; FDR $<0.01$; two-tailed Student's $t$-test followed by Storey's $q$-value) induced at both transcriptome and proteome levels in planta at $6 \mathrm{hpi}$ compared with in KB medium (Fig. 5a). GO analysis showed that 'pathogenesis-related process' was enriched among these mRNAs/ proteins (Fig. 5a), indicating that Pto transcriptionally activates pathogenesis programmes following plant infection. On the other hand, there were cases where expression of mRNAs and proteins was discordant. Interestingly, more proteins were downregulated (168 proteins) than upregulated (39 proteins) in a protein-specific manner (Fig. $5 \mathrm{a}$ ). This may be explained by a prominent role of protein degradation or translation inhibition in bacteria in planta (Fig. 5a). GO analysis showed that 'cell wall biogenesis'-related proteins were suppressed only at the protein level (Fig. 5a). In contrast, more mRNAs were upregulated (207 proteins) than downregulated (49 proteins) in an mRNA-specific manner (Fig. 5a). This implies that the upregulation of specific mRNAs is a key response of Pto at an early stage of infection, and that the induction of mRNAs is not yet reflected in protein abundance at this point. We also compared fold changes in the transcriptome and proteome profiles between pad4 sid 2 and Col- 0 at $48 \mathrm{hpi}$. GO enrichment analysis showed that bacterial processes related to 'chemotaxis' were highly expressed in pad4 sid2 at the protein level, but not at the mRNA level (Fig. 5b). This suggests that chemotaxis-related processes are suppressed by plant SA-mediated immunity at the protein level, and this may be important for bacterial growth inhibition. Collectively, genome-wide comparisons between mRNA and protein expression illuminate the multifaceted control of bacterial gene expression in planta.

Component-specific suppression of the type III secretion system by plant SA pathways. Gene Ontology enrichment analysis showed that the plant SA pathway suppresses a substantial number of bacterial proteins related to pathogenesis, including those comprising the type III secretion system (T3SS) (Fig. 2d). The T3SS is an essential component by which Pto translocates effectors into plant cells to subvert plant immunity and become virulent ${ }^{17}$. We found that the impact of SA pathways was apparent in proteins comprising the tip of the T3SS, namely HrpZ, HrpK and HrpW (Fig. 6a). This suggests that SA pathways target the tip of bacterial T3SS. To confirm this observation, we performed immunoblotting using protein samples directly extracted from infected leaves without physical bacterial isolation. Since bacterial population varies among samples, protein loading was normalized by expression of HrcC protein, which showed a high and consistent level of expression across conditions in the proteome data. HrpZ accumulated in pad4 sid2 plants more than in Col-0 plants, indicating the differential effect of SA pathways on different components of T3SS (Fig. 6b). This also suggests that the bacterial isolation process did not introduce artefacts in the proteome data. To test whether differential expression of HrpZ is due to different bacterial populations in plants, we compared HrpZ protein abundance between Col- 0 at 48 hpi and pad4 sid 2 at $24 \mathrm{hpi}$, time points at which bacterial population densities were comparable (Extended Data Fig. 3c). In this comparison, HrpZ protein accumulated to higher levels in pad4 sid2 than in Col-0 (Fig. 3c), suggesting that bacterial population does not solely explain differences in HrpZ protein expression and thus that SA-mediated immunity may directly target this protein. Relative mRNA expression ( $h r p Z / h r c C)$ was marginally increased in pad4 sid 2 compared with Col-0 at 48 hpi (Fig. 6d), which may partially explain the difference in protein accumulation. In the RNA-seq data, both $h r c C$ and $h r p Z$ expression was higher in pad4 sid 2 compared with Col-0, but $h r p Z / h r c C$ was comparable (1.08-fold difference) between Col-0 and pad4 sid2 (Extended Data Fig. 5). Taken together, we found that accumulation of proteins comprising the tip component of T3SS was suppressed by SA pathways. The relative contributions of transcriptional and post-transcriptional events to this phenomenon remain elusive.

Gene co-expression analysis predicts bacterial gene regulatory logic. Despite the distinct regulation of certain specific mRNAs and proteins, the overall moderate correlation between transcriptome and proteome patterns of Pto suggests that mRNA expression can be a good indicator of bacterial functional expression in planta. Also, bacterial transcriptome analysis is more sensitive than proteome analysis (Fig. 1c) and has access to more diverse datasets from a previous study ${ }^{3}$. Therefore, we reasoned that investigating the regulatory network governing bacterial mRNA expression would help deepen our understanding of bacterial functional regulation. To deconvolute the gene regulatory network of Pto, we used 125 transcriptome datasets of Pto profiled in 38 conditions (generated in a previous study ${ }^{3}$ and in this study). A correlation matrix of 4,765 genes revealed highly correlated gene clusters (Extended Data Fig. 6a), some of which were enriched with known functions (Fig. 7a). Next, we built a gene co-expression network based on the correlation scores and annotated genes with known functions; this allowed us to conclude that genes sharing the same functions tend to be co-expressed (Fig. 7b). For instance, genes related to pathogenesis (mostly T3SS and effector genes), flagellum and iron-starvation responses were found in separate and highly co-expressed gene clusters (Fig. 7a,b). Intriguingly, genes involved in coronatine and alginate biosynthesis were clustered very closely together, implying that these processes might share the same regulatory mechanism (Extended Data Fig. 6b). On the other hand, genes related to coronatine biosynthesis and the T3SS were only mildly correlated with each other (Extended Data Fig. 6b), although it has been shown that expression of corR, the master regulator of coronatine biosynthesis genes, is dependent on $\mathrm{HrpL}$, the master regulator of the T3SS ${ }^{18}$. This suggests that there might be additional regulators that govern the expression patterns of genes related to coronatine biosynthesis and the T3SS. We also found that some genes annotated as effectors were not co-expressed with the majority of effectors (Extended Data Fig. 6b), suggesting that either they function in different contexts or they do not function as effectors. Strong anti-correlation was observed between 'siderophore transport' genes, which are iron-repressive, and 'ferric iron-binding' genes, which are involved in iron-inducible bacterioferritin (Fig. $7 \mathrm{c}$ ), indicating that this analysis could capture known expression patterns. 


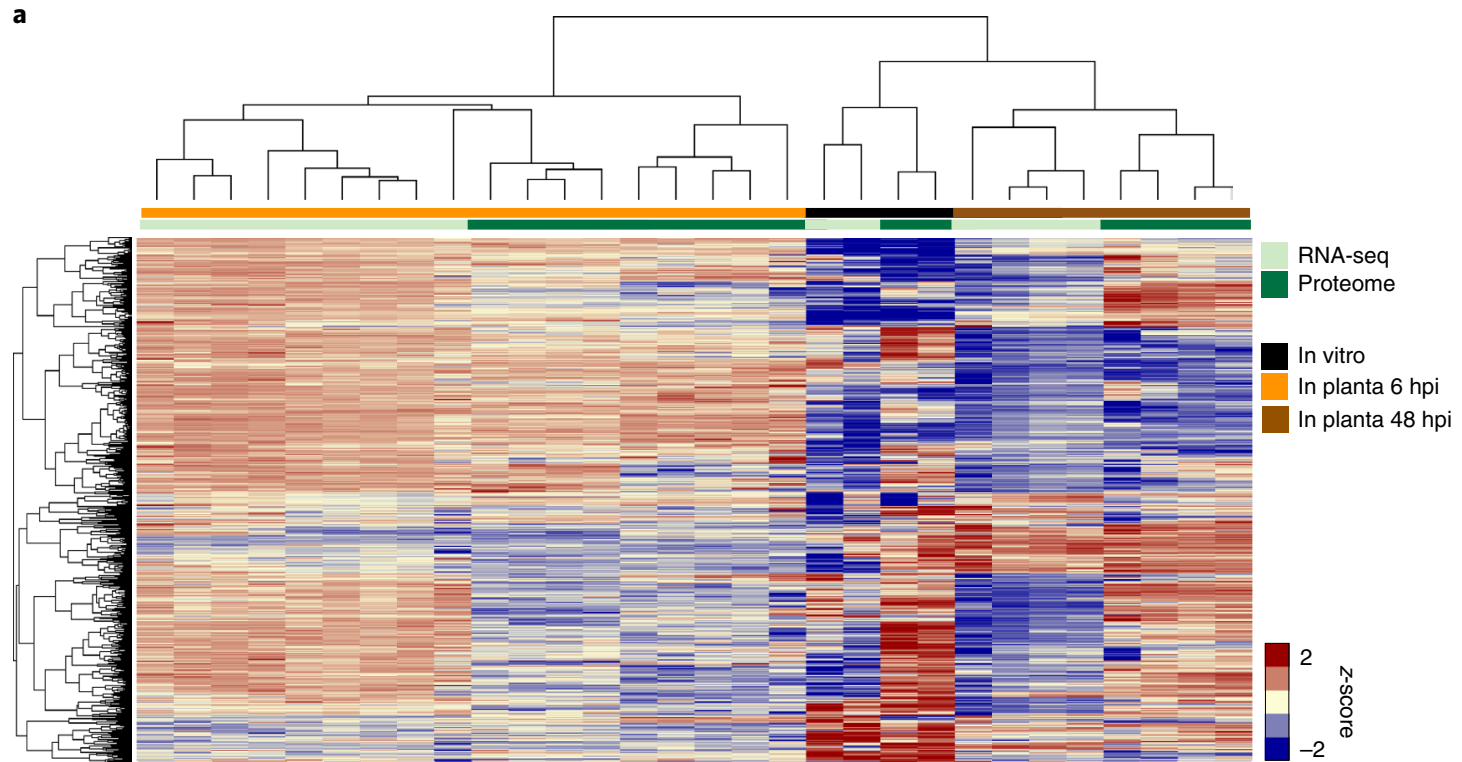

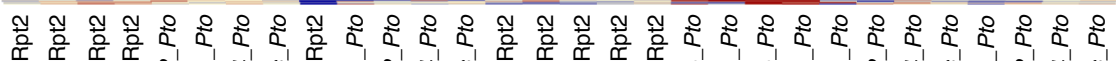

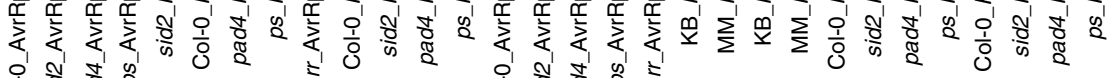

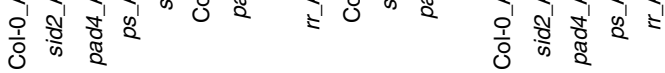

b

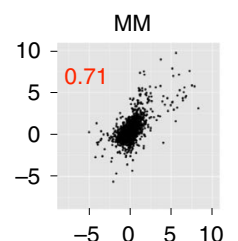

Col-0 AvrRpt2 $6 \mathrm{~h}$
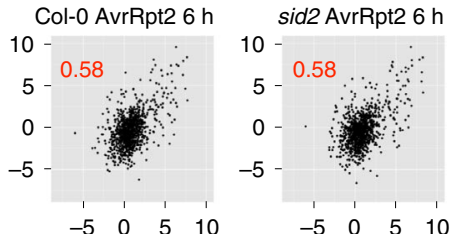

pad4 AvrRpt2 $6 \mathrm{~h}$ ps AvrRpt2 $6 \mathrm{~h}$ rr AvrRpt2 $6 \mathrm{~h}$ Col-0 Pto
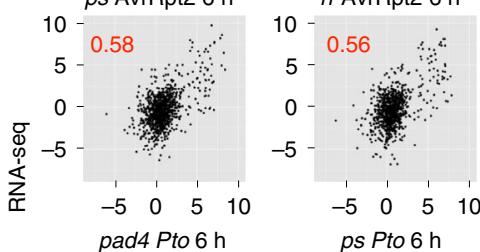

$10-\mathrm{Col}-0$
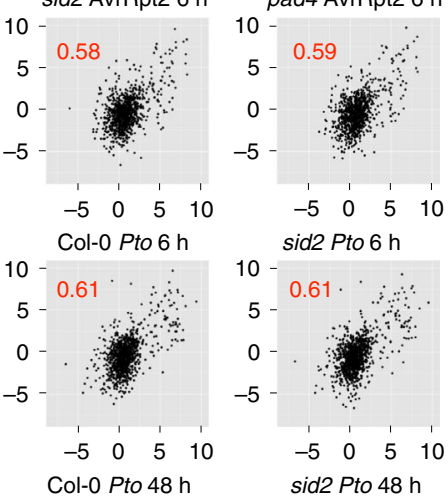

sid2 Pto $6 \mathrm{~h}$

$10-$

$10-0.61$
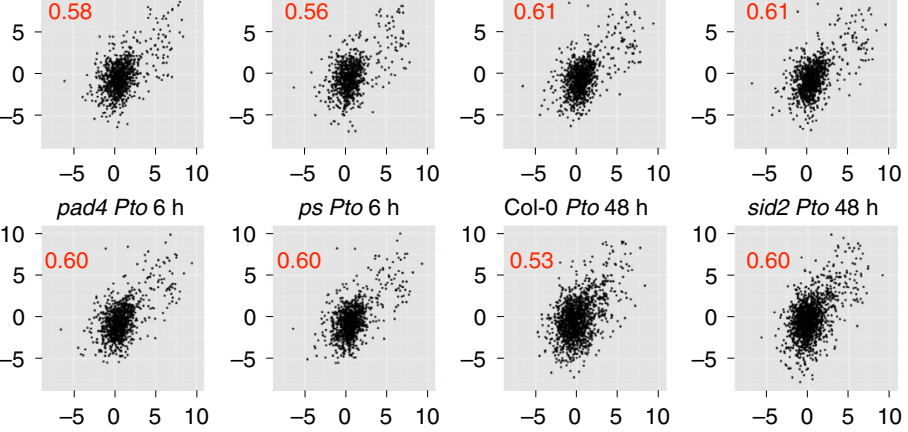

sid2 Pto $48 \mathrm{~h}$
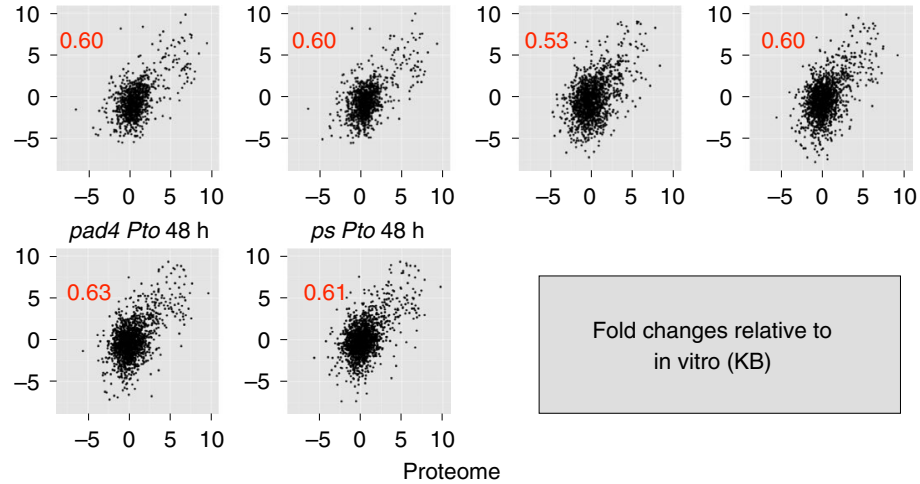

Fold changes relative to in vitro (KB)

Fig. 4 | Conserved and divergent regulation of mRNA and protein expression in Pto. a, Transcriptome and proteome data were separately standardized using $\log _{2}$ [z-scores] and combined, followed by hierarchical clustering. mRNAs/proteins detected in all conditions were subject to analysis. Light and dark green sidebars represent transcriptome and proteome data, respectively. Black, orange and brown sidebars represent in vitro (KB), in planta 6 hpi and in planta $48 \mathrm{hpi}$, respectively. MM, minimal medium; ps, pad4 sid2; rr, rpm1 rps2. b. Comparisons between transcriptome and proteome data under each condition. Fold changes relative to in vitro (KB) conditions were subjected to analysis. Pearson's correlation coefficients are shown. mRNAs/proteins detected in both the transcriptome and proteome in each comparison were used for this analysis. For a,b, see Supplementary Data 13 for sample sizes.

We anticipated that groups of highly co-expressed genes contain transcriptional regulators (TRs) and their targets. Indeed, $h r p L$ and the iron-starvation sigma factor $p v d S$ were co-expressed with their targets, T3SS genes and iron-starvation genes, respectively (Fig. 6b). To test whether gene co-expression data can predict gene regulatory hierarchy, we selected three putative TRs, PSPTO_0384, 


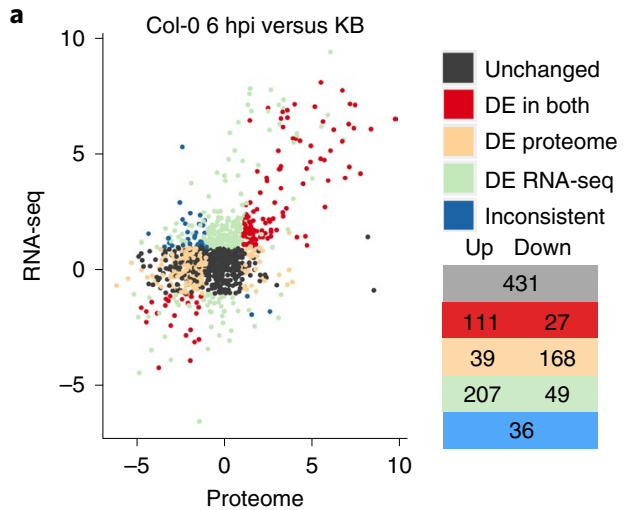

\begin{tabular}{|clcc|}
\hline Category & \multicolumn{1}{c}{ GO term } & \multicolumn{1}{c}{$P$ value } & Fold enriched \\
\hline \multirow{2}{*}{ Both up } & Pathogenesis & $8.85 \times 10^{-15}$ & 13.1 \\
& Translation & $1.94 \times 10^{-12}$ & 9.0 \\
& Alginic acid metabolic & $6.32 \times 10^{-12}$ & 32.6 \\
\hline $\begin{array}{c}\text { Proteome } \\
\text { down }\end{array}$ & Cell wall biogenesis & $3.42 \times 10^{-03}$ & 10.2 \\
\hline
\end{tabular}

b

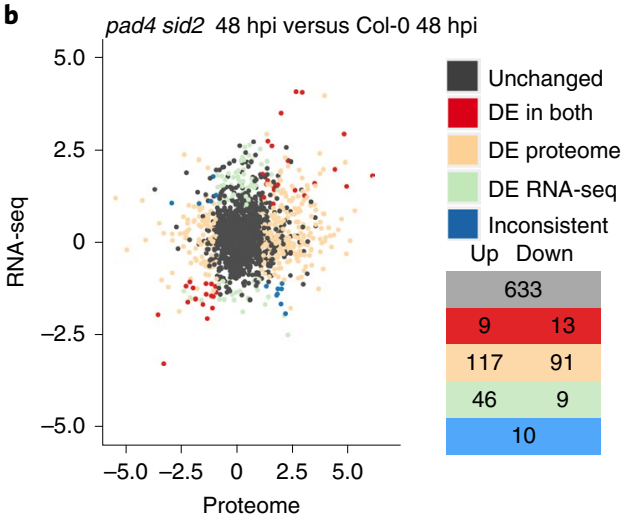

\begin{tabular}{|clcc|}
\hline Category & \multicolumn{1}{c}{ GO term } & \multicolumn{1}{c|}{$P$ value } & Fold enriched \\
\hline & Carboxylic acid metabolic & $1.90 \times 10^{-04}$ & 2.4 \\
$\begin{array}{c}\text { Proteome } \\
\text { up }\end{array}$ & Chemotaxis & $4.26 \times 10^{-03}$ & 6.8 \\
& Nucleobase & $5.35 \times 10^{-03}$ & 3.3 \\
& Amine metabolic & $6.93 \times 10^{-03}$ & 2.2 \\
$\begin{array}{c}\text { Proteome } \\
\text { down }\end{array}$ & Translation & $2.69 \times 10^{-05}$ & 6.4 \\
\hline
\end{tabular}

Fig. 5 | Bacterial functions showing concordant and discordant regulation at the mRNA and protein levels. a, mRNA/protein expression fold changes between in planta (Col-0) at $6 \mathrm{hpi}$ and in vitro (KB) were compared. mRNAs/proteins differentially expressed $\left(D E ;\left|\log _{2}[F C]\right|>1 ; F D R<0.01 ; t\right.$ two-tailed Student's $t$-test followed by Storey's $q$-value) in both, either and neither (unchanged) transcriptome and proteome studies were grouped and colour-coded. mRNAs/proteins differentially expressed in the opposite direction are coloured blue (inconsistent). The numbers of mRNAs/proteins are shown for each category. List of GO terms enriched in the group of proteins that are significantly induced in planta at both mRNA and protein levels, or proteins that are significantly suppressed in planta only at the protein level. b, mRNA/protein expression fold changes between Col-0 and pad 4 sid2 at $48 \mathrm{hpi}$ were compared. List of GO terms enriched in the group of proteins that are significantly induced in planta at both mRNA and protein levels, or proteins that are significantly suppressed in planta only at the protein level ( $\left|\log _{2}[F C]\right|>1 ; F D R<0.01$; two-tailed Student's $t$-test followed by Storey's $q$-value). For $\mathbf{a}, \mathbf{b}$, see Supplementary Data 8 for the full gene list and GO list. mRNAs/proteins detected in both the transcriptome and proteome in each comparison were used for these analyses. For $\mathbf{a}, \mathbf{b}$, see Supplementary Data 13 for sample sizes.

PSPTO_3050 and PSPTO_3467, whose functions have not been previously characterized. These TRs were selected from three clusters of highly correlated genes, with PSPTO_0384 and PSPTO_3467 being the only genes annotated as putative TR and PSPTO_3050 being randomly picked from two TRs in the cluster (Supplementary Data 10). We generated Pto strains that overexpress each of the TRs. We then analysed the expression of predicted target genes that were highly co-expressed with individual TRs. Remarkably, for the three TRs, all or most of the predicted target genes were highly expressed in the TR overexpression lines in vitro (Fig. 8a), supporting the predicted regulatory hierarchy. This was further confirmed in planta at 6hpi for PSPTO_3050, but overexpression of PSPTO_0384 or PSPTO_4908 induced only a small number of the predicted targets (Fig. 8b). This is probably explained by the fact that genes highly co-expressed with these two TRs are already strongly induced in wild-type Pto in planta at 6 hpi (Fig. 8c) and, thus, overexpression of these TRs did not lead to further induction of the predicted target genes. Notably, all the TR-overexpressing Pto grew significantly (adjusted $P<0.01$; two-tailed Student's $t$-test followed by Benjamini-Hochberg method) better than wild-type Pto in Col-0 plants (Fig. 8d), suggesting that the three TRs and some of their regulons play positive roles in Pto growth in plants. Collectively, in planta transcriptome data of Pto under diverse conditions enable gene co-expression analysis that can be used to identify previously unknown bacterial gene clusters contributing to bacterial growth in planta, as well as to reveal the regulatory logic in the gene clusters.

\section{Discussion}

In this study, we analysed the transcriptome and proteome of the bacterial pathogen Pto both in vitro and in planta under various conditions. Integrative multi-omics analysis revealed that bacterial mRNA and protein expression were moderately correlated in both liquid media and resistant and susceptible plants (Fig. 4). Our data indicate that changes in bacterial transcriptomes can serve as a reliable predictor of the proteome changes elicited by plant colonization. Previous studies using plants, yeasts and mammals showed varying degrees of correlation between transcriptomes and proteomes but, in most cases, the correlation was considerably lower than for the bacterial pathogen Pto shown in this study ${ }^{19-23}$. This suggests that in Pto, at the population level, mRNAs are faithfully translated into proteins in various situations in planta.

Multi-modal measurements of bacterial responses during infection provide a systematic view of bacterial gene regulation that cannot be captured by analysis of any single modality alone. By analysis of cases in which mRNA and protein expression do not correlate with each other, we found that bacterial cell wall biogenesis-related function was specifically suppressed at the protein level in Pto at an early stage of infection (Fig. 5a). Most of these proteins were 

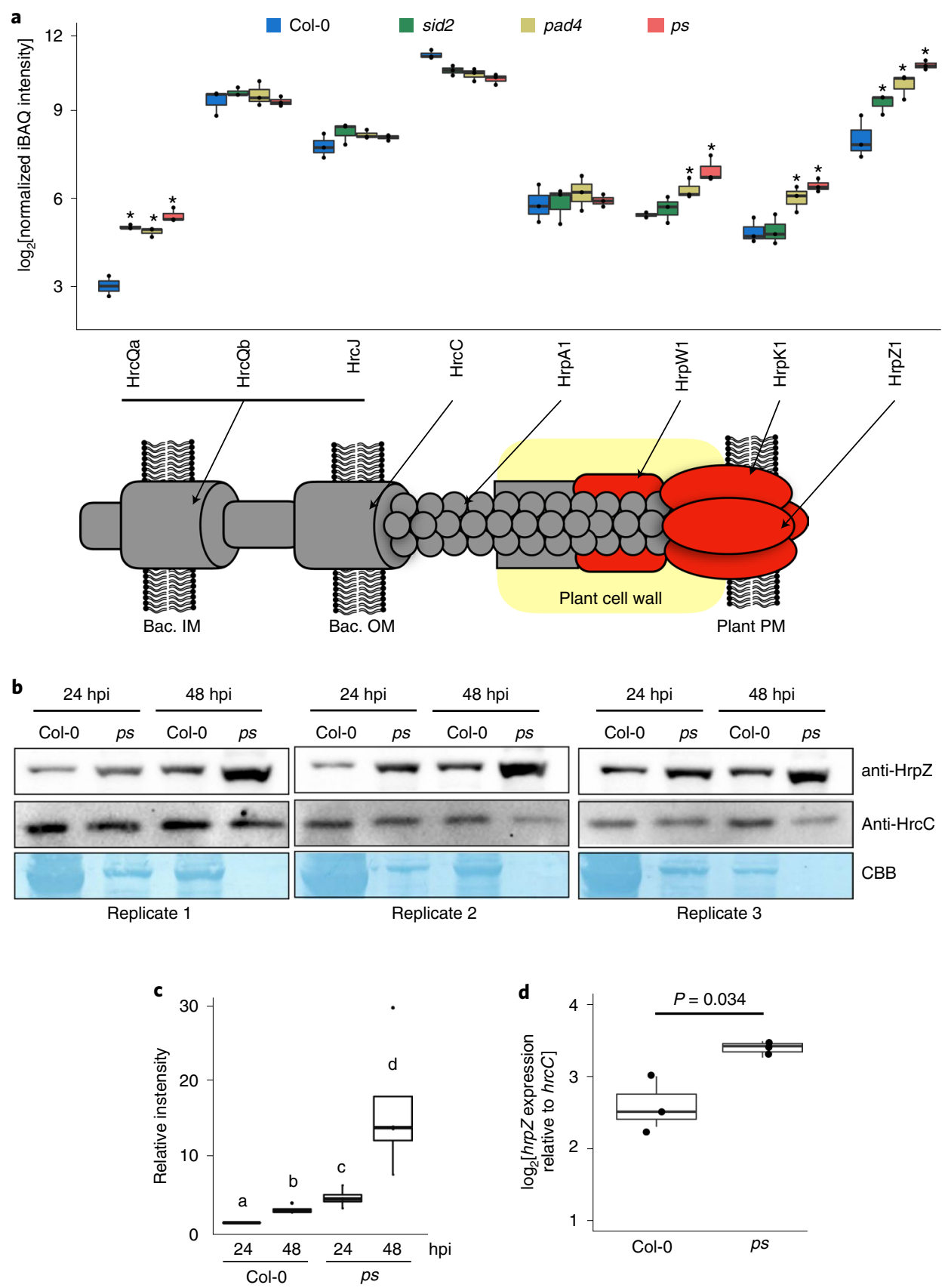

Fig. 6 | Component-specific suppression of the type III secretion system by plant SA pathways. a, Expression of T3SS-related proteins based on proteome data (normalized $\mathrm{BAQ}$ value). Approximate localization of each protein is shown in the diagram. Asterisks indicate statistically significant differences compared with Col-0 (adjusted $P<0.05$; two-tailed Student's $t$-test followed by Benjamini-Hochberg method). Bac. IM, bacterial inner membrane; Bac. OM, bacterial outer membrane; plant PM, plant plasma membrane. See Supplementary Data 13 for sample sizes. b, HrpZ and HrcC proteins detected by immunoblotting using specific antibodies. Protein loading amount was adjusted relative to the expression of the Hrc $\mathrm{C}$ protein. The experiments were repeated three times with similar results. CBB, Coomassie Brilliant Blue staining. c, Relative intensity of HrpZ expression normalized to $\mathrm{HrcC}$ expression based on the data in $\mathbf{b}$. Different letters indicate statistically significant differences (adjusted $P<0.05$; two-tailed Student's $t$-test followed by Benjamini-Hochberg method). In b,c, plants were infiltrated with Pto at $\mathrm{OD}_{600}=0.005$ and harvested at 24 and 48 hpi. d, RT-qPCR analysis of log 2 [hrpZ relative to $\mathrm{hrcC}$ ]. Plants were infiltrated with $P$ to at $\mathrm{OD}_{600}=0.005$ and harvested at $48 \mathrm{hpi}$. The $P$ value (two-tailed Student's $t$-test) is indicated. In $\mathbf{c}, \mathbf{d}$, $n=3$ biological replicates from three independent experiments. In a,c,d, results are shown as box plots with boxes displaying the 25th-75th percentiles, the centre line indicating the median and whiskers extending to the minimum and maximum values no further than $1.5 \times$ interquartile range.

enzymes that probably catalyse peptidoglycan biosynthesis. Because these proteins localize in the cytosol, it is likely that suppression of these proteins in planta is mediated by bacteria themselves and may be an adaptive trait for them, because Pto is a virulent pathogen. By comparison of Pto in Col-0 and pad4 sid2 at $48 \mathrm{hpi}$, we found that chemotaxis-related function was suppressed by the SA pathways of $A$. thaliana at the protein level (Fig. 5b). Since chemotaxis is required for virulence of certain plant bacterial pathogens ${ }^{24}$, suppression of bacterial motility at the protein level may be a part of plant defence strategy. It is possible that bacterial quorum sensing ${ }^{25}$ 


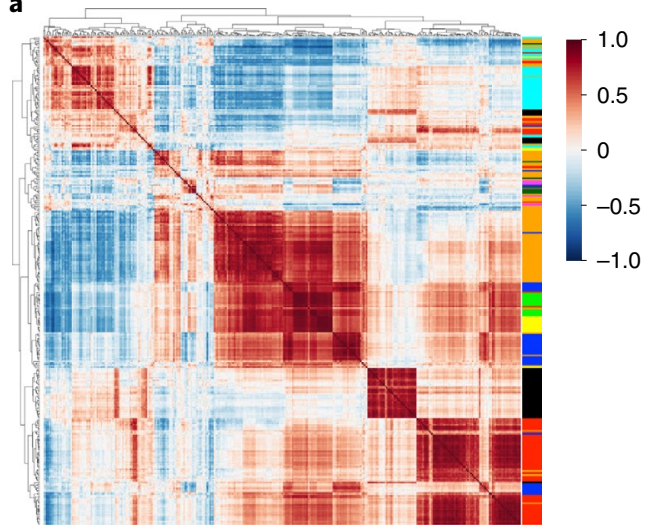

C

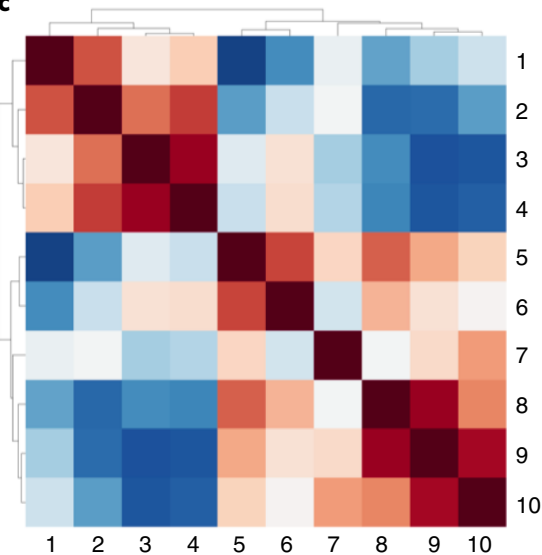

(1) Ferric iron binding

(2) Siderophore transport

(3) Chemotaxis

(4) Flagellum assembly

(5) Phosphorelay sensor kinase

(9) ATP synth. prot
(10) Translation
(6) ATPase activity

(7) Cell division

(8) Acetyl-CoA carboxylase

(9) ATP synth. proton transport

Alginate

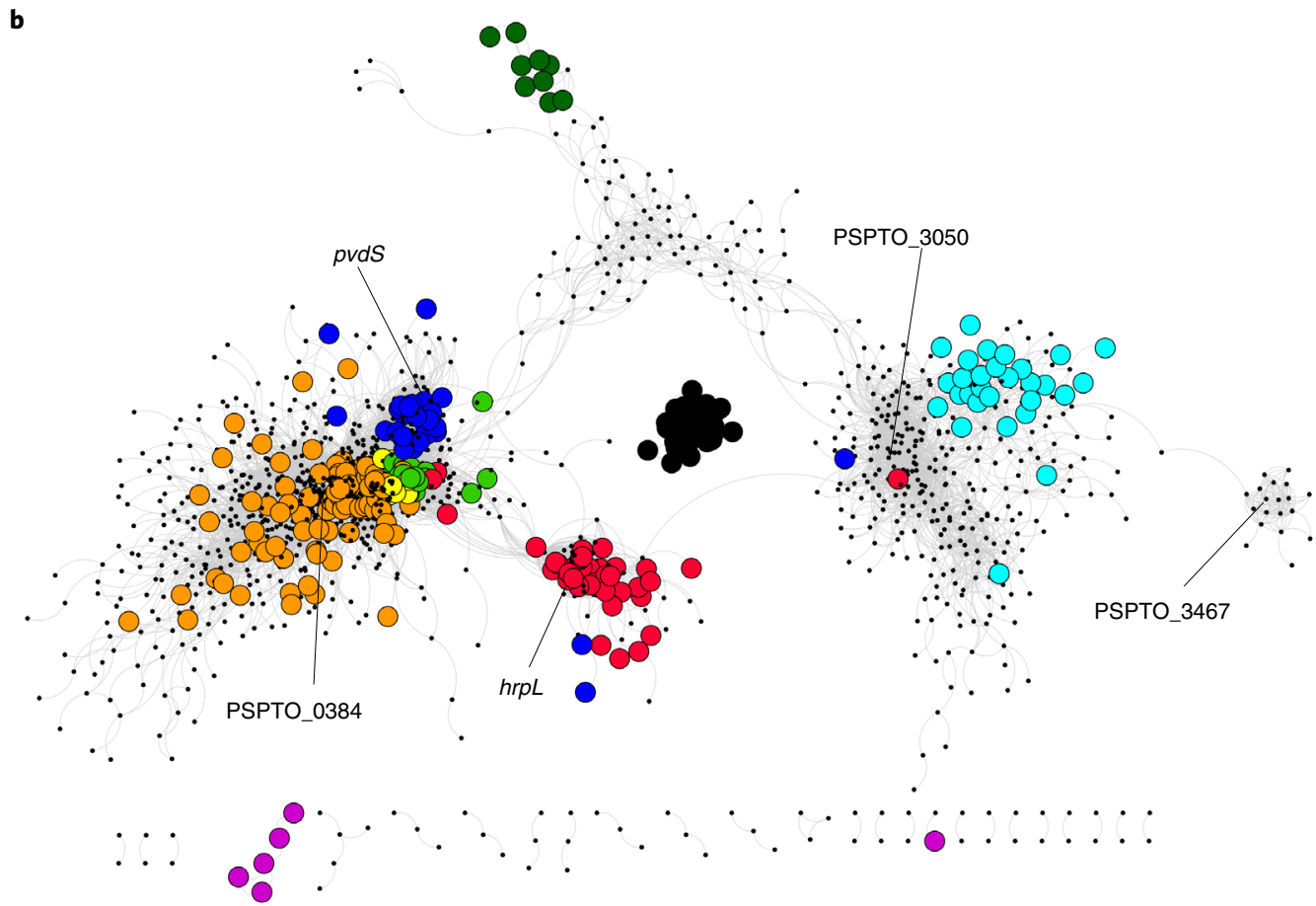

Fig. 7 | mRNA co-expression analysis of Pto reveals functional mRNA modules. a, The correlation matrix of selected Pto mRNAs (330 mRNAs), with functional annotations shown in the sidebar. For the full correlation matrix, see Extended Data Fig. 6a. For the full dataset, see Supplementary Data 9. b, mRNA co-expression network of Pto. Each node represents a gene. Highly correlated $\left(R^{2}>0.8\right)$ genes are connected with edges. Functions are colour-coded. c, The correlation matrix of bacterial GO expression; selected GOs are shown. In a-c, 125 transcriptome datasets of Pto profiled under 38 conditions (generated in a previous study ${ }^{3}$ and in this study) were used. Pearson's correlation coefficients were used.

may explain plant genotype effects observed at $48 \mathrm{hpi}$, because bacterial population density was higher in SA mutants compared with Col-0 at this time point.

We found that protein expression of the tip component of the T3SS was suppressed by the SA pathway of $A$. thaliana, while most of the other components of the T3SS were not affected (Fig. 6a,b). These tip proteins, HrpZ1, HrpW1 and HrpK, were shown to function redundantly in effector translocation ${ }^{26}$, and lack of $h r p K$ per se compromised the growth of Pto in A. thaliana ${ }^{27}$. Thus, targeting these proteins is sufficient to dampen the virulence of Pto. Our results suggest that SA-mediated plant immunity may promote the degradation of these proteins to suppress effector translocation into plant cells and thus to inhibit pathogen growth, although we do not exclude the contribution of transcriptional regulation. It is tempting to speculate that plant immunity directly targets the tip component of the T3SS at the plant cell wall. Because the T3SS must cross the cell wall to successfully translocate effectors into plant cells, it therefore represents an ideal target for plants to counter bacterial virulence. We have recently shown that plant-secreted proteases cleave an evolutionary conserved bacterial protein to inhibit growth of $\mathrm{Pto}^{28}$. Screening of immune-activated, plant-secreted proteases or chemicals for their ability to cleave T3SS components could 
a

PSPTO_0384

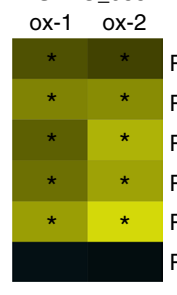

PSPTO_0384
PSPTO_0001
PSPTO_3953
PSPTO_0655
PSPTO_0763
PSPTO_5315

b

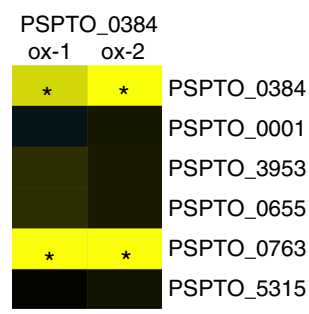

In vitro

PSPTO_3050

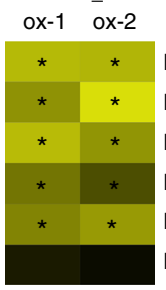

PSPTO_3050

PSPTO_3355

PSPTO_2310

PSPTO_1062

PSPTO_4540

PSPTO_5315

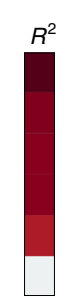

PSPTO_3467

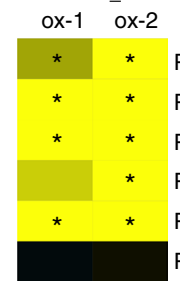

PSPTO_3467
PSPTO_5198
PSPTO_0307
PSPTO_3466
PSPTO_5315
PSPTO_1062
In planta

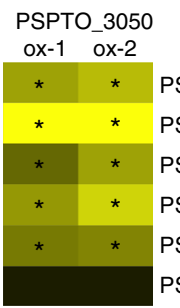

PSPTO_3050
PSPTO_3355
PSPTO_2310
PSPTO_1062
PSPTO_4540
PSPTO_5315

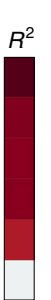

PSPTO_3467

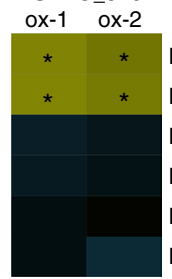

PSTO_3467

PSPTO_5198

PSPTO_0307

PSPTO_3466

PSPTO_5315

PSPTO 1062

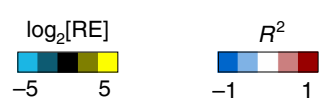

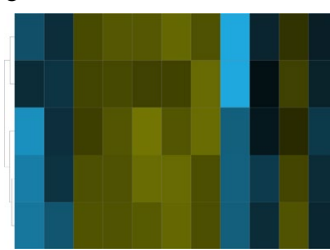

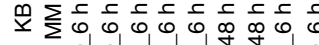

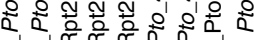

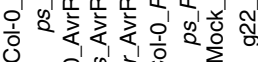
옹

d

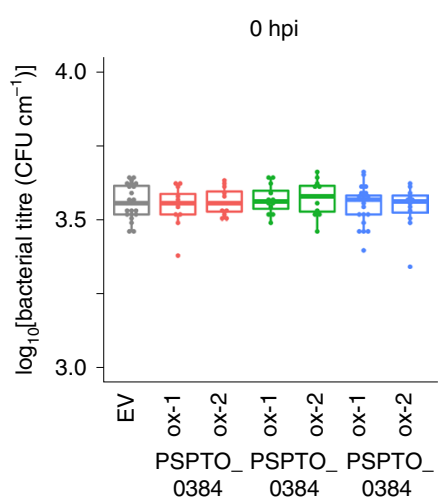

PSPTO_0384
PSPTO_0763
PSPTO_0001
PSPTO_0655
PSPTO_3953

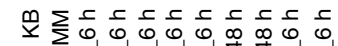

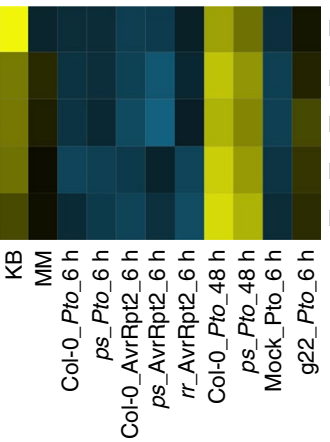

48 hpi

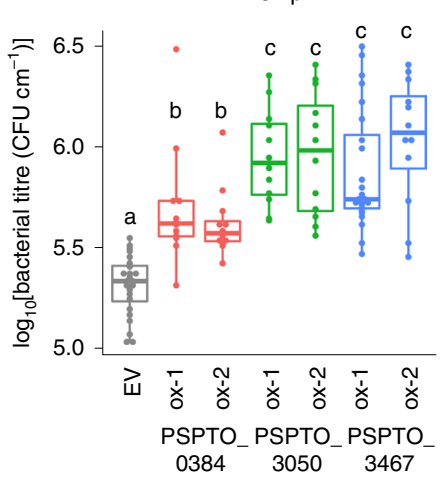

PSPTO_2310
PSPTO_1062
PSPTO_3355
PSPTO_3050
PSPTO 4540

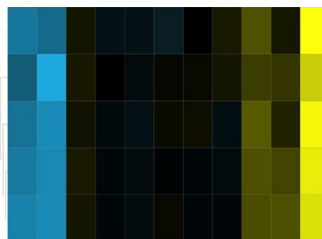

PSPTO_0307

PSPTO_3466

PSPTO_5198

PSPTO_3467

PSPTO_5315

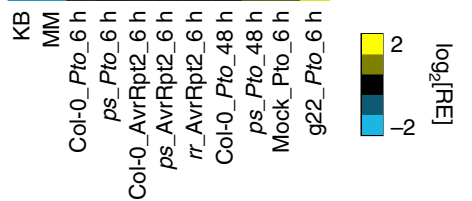

48 hpi

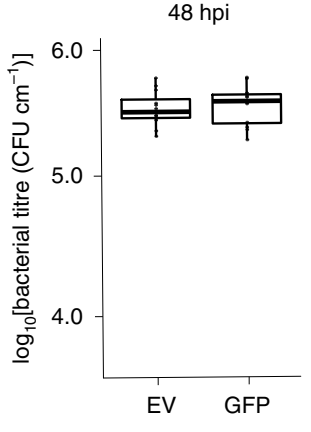

Fig. 8 | The roles of transcriptional regulators found in bacterial gene regulatory modules. a,b, Two independent Pto strains overexpressing putative TRs were generated (ox-1 and ox-2). RT-qPCR analysis of in vitro (a) and in planta (b) mRNA expression fold changes between Pto overexpressing putative TRs and wild-type Pto. Asterisks indicate significant differences ( $\left|\log _{2}[F C]\right|>1, P<0.05$; two-tailed Student's $t$-test). Pearson's correlation coefficients between each mRNA and TRs are shown in the sidebars on the right. The bottom genes showed low correlation $\left(-0.2<R^{2}<0.2\right)$ with each TR, while the other genes showed high correlation $\left(R^{2}>0.7\right) . n=3$ biological replicates from three independent experiments. See Supplementary Data 11 for the numerical value of $R^{2}$. c, Relative expression (RE) of TRs and genes highly co-expressed with each TR. See Supplementary Data 13 for sample sizes. MM, minimal medium. d, Growth of Pto overexpressing putative TRs or an empty vector (EV) (infiltrated at $\mathrm{OD}_{600}=0.001$ ) in Col- 0 at $0 \mathrm{hpi}$ (left) and $48 \mathrm{hpi}$ (middle). Right: growth of wild-type Pto and Pto overexpressing green fluorescent protein (GFP; infiltrated at $\mathrm{OD}_{600}=0.001$ ) in Col-0 at $48 \mathrm{hpi}$. $n=12$ biological replicates from three independent experiments. Different letters indicate statistically significant differences (adjusted $P<0.01$; two-tailed Student's $t$-test followed by Benjamini-Hochberg method). Results are shown as box plots with boxes displaying the 25th-75th percentiles, the centre line indicating the median and whiskers extending to the minimum and maximum values no further than $1.5 \times$ interquartile range. 
potentially lead to the discovery of how plants target this component that is evolutionarily conserved and essential for virulence.

Messenger RNA co-expression analysis identified groups of highly co-expressed bacterial mRNAs with both known and unknown functions. Using mRNA co-expression data, we could predict three pairs of putative TRs and mRNAs whose expression is affected by these TRs (Fig. 8a). The TRs and their potential target mRNAs are induced in planta at an early and/or a late time point of infection (Fig. 8c), and overexpression of TRs led to enhanced bacterial growth in planta (Fig. $8 \mathrm{~d}$ ). Therefore, our approach has the capability to identify previously unknown gene modules that are important for virulence in plants. mRNAs co-expressed with the TR PSPTO_0384 are induced in susceptible plants and suppressed in those that have engaged PTI (flg22 pre-treatment; Fig. 8c), which is similar to known virulence-related genes such as the T3SS and effectors ${ }^{3}$. Thus, the induction of these mRNAs might be important for virulence and this could explain the enhanced growth of PSPTO_0384-ox strains in planta (Fig. 8d). Interestingly, mRNAs co-expressed with either of the other two TRs, PSPTO_0350 and PSPTO_3467, were induced by PTI activation while overexpression of these TRs led to enhanced bacterial growth (Fig. 8d). It is possible that these genes are involved in stress adaptation and contribute to bacterial growth in plants. Previously, we observed that PTI activation induces a number of Pto mRNAs ( 800 mRNAs) whose functions are not well understood ${ }^{3}$. Investigating such genes might help us identify previously uncharacterized genes related to bacterial stress adaptation and/or virulence in plants. Taken together, in planta bacterial multi-omics represents a new strategy for studying the molecular mechanisms underlying bacterial virulence and plant immunity.

\section{Methods}

Plant materials and growth conditions. The $A$. thaliana accession Col- 0 was the background of all $A$. thaliana mutants used in this study. A. thaliana mutants rpm1-3 rps2-101C (ref. ${ }^{29}$ ), pad4-1 (ref. ${ }^{30}$ ), sid2-2 (ref. ${ }^{31}$ ) and pad4 sid2 (ref. ${ }^{6}$ ) were described previously. Plants were grown in a chamber at $22^{\circ} \mathrm{C}$ with a 10 -h light period and $60 \%$ relative humidity for $24 \mathrm{~d}$, and then in another chamber at $22^{\circ} \mathrm{C}$ with a 12 -h light period and $60 \%$ relative humidity. Different genotypes were mixed in a pot to randomize the growth condition. For all experiments, 31- to 33-day-old plants were used.

Bacterial strains. Pto DC3000 carrying an empty vector (pLAFR) or avrRpt2 expressed from $\mathrm{pLAFR}^{32}$ were described previously. The Pto overexpression strains were generated as previously described ${ }^{33}$. The coding sequences of GFP, PSPTO_0384, PSPTO_3050 and PSPTO_3467 were amplified by PCR, cloned into the pENTR/D-TOPO vector and then transferred into pCPP5040 (gentamicin-resistant) by the LR reaction. The Pto overexpression strains were generated by a tri-parental mating of the Pto wild-type strain, E. coli carrying each construct and an E. coli strain carrying pRK2013 (kanamycin-resistant). The transformed Pto strains were selected with $50 \mu \mathrm{g} \mathrm{ml}^{-1}$ rifampicin, $5 \mu \mathrm{g} \mathrm{ml}^{-1}$ gentamicin and $50 \mu \mathrm{g} \mathrm{ml}^{-1}$ kanamycin.

Accession numbers. The accession numbers for the genes discussed in this article are as follows: AtPAD4 (AT3G52430), AtSID2 (AT1G74710), AtRPS2 (AT3G03600), AtRPM1 (AT3G07040), hrcC (PSPTO_1389) and $h r p Z$ (PSPTO_1382).

Preparation of in vitro bacterial samples. Bacteria were grown in either $\mathrm{KB}$ or

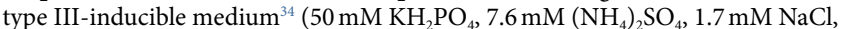
$1.7 \mathrm{mM} \mathrm{MgCl}_{2}$ and $10 \mathrm{mM}$ fructose) at $28^{\circ} \mathrm{C}$ until they reached $\mathrm{OD}_{600}=0.65$ (exponential phase). Following harvesting of the bacterial culture, 0.1 volumes of $5 \%$ phenol and $95 \%$ ethanol were added. The culture was then centrifuged to harvest the bacterial pellet, followed by total RNA and/or protein extraction.

Bacterial infection of plant leaves and sampling. Pto strains were cultured in $\mathrm{KB}$ at $28^{\circ} \mathrm{C}$ at 200 r.p.m. Bacteria were harvested by centrifugation and resuspended in sterile water to $\mathrm{OD}_{600}=0.5\left(\sim 2.5 \times 10^{8}\right.$ colony-forming units (c.f.u.) $\left.\mathrm{ml}^{-1}\right)$ and $\mathrm{OD}_{600}=0.005\left(\sim 2.5 \times 10^{6}\right.$ c.f.u. $\left.\mathrm{ml}^{-1}\right)$ for harvesting at 6 and 48 hpi, respectively. In total, $80-100 \mathrm{~A}$. thaliana leaves (four leaves per plant) were syringe-inoculated with bacterial suspension using a needleless syringe. For each biological replicate, the same bacterial suspension was used for different genotypes and infiltration was randomized. The infected leaves were harvested at 6 or 48 hpi. Sampling was conducted for each plant genotype separately, requiring approximately $5 \mathrm{~min}$ per genotype. Leaves were immediately frozen in liquid nitrogen and stored at $-80^{\circ} \mathrm{C}$. The bacterial growth assay was performed as described before ${ }^{35}$.

In planta bacterial transcriptomics. Sample preparation and RNA-seq. In planta bacterial transcriptome analysis was conducted as described previously ${ }^{36}$. Briefly, bacteria-infected leaves were coarsely pulverized and released in the bacterial isolation buffer ( $9.5 \%$ ethanol, $0.5 \%$ phenol and $25 \mathrm{mM}$ tris(2-carboxyethyl) phosphine) $\mathrm{pH} 4.5$ adjusted with $\mathrm{NaOH}$ ) at $4{ }^{\circ} \mathrm{C}$, filtered and centrifuged to isolate bacterial from plant cells. RNA was isolated from bacterial cells and ribosomal RNA was depleted to enrich mRNA, and complementary DNA libraries were prepared. The cDNA libraries were sequenced using an Illumina HiSeq 3000 system with a 150-base pair, strand-specific, single-end read, resulting in $\sim 10$ million reads per sample. The resulting reads were mapped onto the Pto DC3000 genome/CDS (Pseudomonas Genome Database) using Bowtie2 (ref. ${ }^{37}$ ). Mapped reads were counted with the Python package HTSeq ${ }^{38}$. The RNA-seq data used in this study are deposited in NCBI Gene Expression Omnibus database (accession no. GSE138901).

Data analysis. Statistical analysis of the RNA-seq data was performed in the Renvironment. Genes with average counts $<5$ were excluded from the analysis. In this filtering, 387 genes whose average count was non-zero were removed. Most of the genes showed very low counts across all samples, except for some showing high expression under a few conditions, namely 48 hpi (Extended Data Fig. 2a). This is probably due to the higher sequence depth of these samples. Because bacterial population is higher at $48 \mathrm{hpi}$ in SA mutants, these samples tend to be sequenced more deeply than other samples. While three of the pad4 sid 2 samples showed consistently high counts, there was no clear pattern in sid2 and pad4, which also showed a marked difference in transcriptome patterns compared with Col-0, suggesting that this count variation is not biological but rather technical (that is, sequence depth), and thus removal of these genes is unlikely to lead to the loss of biologically relevant information for further analyses. The count data were normalized and log-transformed by the function calcNormFactors (trimmed mean of $M$-values (TMM) normalization) in the package edgeR and the function voomWithQualityWeights in the package limma, respectively. To each gene, a linear model was fitted using the function lmFit in the limma package with the following terms: Sgtr $=\mathrm{GTgt}+R \mathrm{r}+$ egtr, where Sgtr is the $\log _{2}$ count per million, GTgt is the host genotype-Pto strain interaction and the random factors, $R r$ is the biological replicate and egtr is the residual. The eBayes function in the limma package was used for variance shrinkage during the calculation of $P$ values. The false discovery rate (FDR; Storey's $q$-values) was calculated using the qvalue function in the qualue package ${ }^{39}$. Genes with $q<0.01$ and $\log _{2}[\mathrm{FC}]>1$ were defined as differentially expressed genes. The prcomp function was used for principal component analysis. Hierarchical clustering was performed using either the dist and hclust functions in the Renvironment or Cluster3.0 software ${ }^{40}$. Heat maps were created with the heatmap3 or pheatmap function in the Renvironment or using TreeView ${ }^{41}$. Enriched GO terms were identified using the BiNGO plugin for Cytoscape ${ }^{42}$. Scatter plots and box plots were generated using the R package ggplot2. Correlation matrices were made by cor function, and the correlation heatmap was drawn by pheatmap in the Renvironment. Gene correlation networks were created in Cytoscape with the yFiles Layout Algorithm.

Gene co-expression analysis. RNA-seq data obtained in a previous study ${ }^{3}$ and the present study were combined (see Supplementary Data 9 for the full dataset). Data were TMM-normalized and voom $\left(\log _{2}\right)$-transformed. Pairwise Pearson's correlation coefficients were calculated. Keywords related to particular functions were searched for on the gene annotation (available at www.pseudomonas.com) to stratify genes for the purpose of visualization (Fig. 7a). Keywords used in this analysis were as follows: 'type III', 'alginate', 'flagellar', 'flagellin', 'flagellum, 'NADH dehydrogenase,' 'coronamic acid', 'coronafacic acid', 'ribosomal protein', 'phosphate transporter' and 'PSPTO_B'. Iron-starvation genes were selected based on a previous study ${ }^{43}$

In planta bacterial proteomics. Bacterial isolation and protein extraction. Bacterial isolation was done as described above. The TriFast solution was mixed with 0.2 volumes of chloroform, and the organic (lower) phase was isolated by centrifugation. The aqueous (upper) phase was isolated for RNA extraction for the simultaneous profiling of transcriptomes and proteomes. The organic phase was mixed with 4 volumes of $\mathrm{MeOH} 0.01 \mathrm{M}$ ammonium acetate and incubated at $-20^{\circ} \mathrm{C}$ overnight to precipitate proteins. The precipitated proteins were washed twice with $\mathrm{MeOH} 0.01 \mathrm{M}$ ammonium acetate and then washed once with $80 \%$ acetone. Proteins were stored in $80 \%$ acetone at $-20^{\circ} \mathrm{C}$.

Sample preparation and fractionation. Proteins were pelleted and re-dissolved

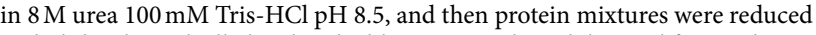
with dithiothreitol, alkylated with chloroacetamide and digested first with Lys-C for $3 \mathrm{~h}$ and subsequently with trypsin overnight. Samples were submitted to styrenedivinylbenzene-reverse phase sulfonated (SDB-RPS) fractionation using a protocol adapted from a previous report ${ }^{44}$. In brief, stage tips were prepared with two layers of SDB-RPS membrane and activated with $100 \mu \mathrm{l}$ of acetonitrile (ACN), followed by equilibration with $100 \mu \mathrm{l}$ of equilibration buffer ( $30 \%$ (v/v) $\mathrm{MeOH}$, 
$1 \%(\mathrm{v} / \mathrm{v})$ trifluoroacetic acid (TFA)) and $100 \mu \mathrm{l}$ of $0.2 \%$ TFA. Next, peptides were immobilized on the membrane and washed with $100 \mu$ l of $0.2 \%$ TFA. Peptides were then eluted into three consecutive fractions using SDB-RPS buffer $1(100 \mathrm{mM}$ $\mathrm{NH}_{4} \mathrm{HCO}_{2}, 40 \%$ (v/v) ACN, 0.5\% FA), SDB-RPS buffer $2\left(150 \mathrm{mM} \mathrm{NH}_{4} \mathrm{HCO}_{2}, 60 \%\right.$ $(\mathrm{v} / \mathrm{v}) \mathrm{ACN}, 0.5 \%$ formic acid) and finally SDB-RPS buffer 3 ( $5 \%$ ammonia (v/v), $80 \%(\mathrm{v} / \mathrm{v}) \mathrm{ACN})$. The collected fractions were evaporated to dryness to remove residual ammonia.

Liquid chromatography-tandem mass spectrometry (LC-MS/MS) data acquisition. Dried peptides were re-dissolved in $2 \% \mathrm{ACN}$ and $0.1 \%$ TFA for analysis and adjusted to a final concentration of $0.1 \mu \mathrm{g} \mathrm{\mu l}^{-1}$. Samples were analysed using either an EASY-nLC 1200 (Thermo Fisher) coupled to a Q Exactive Plus mass spectrometer (Thermo Fisher) or an EASY-nLC 1000 (Thermo Fisher) coupled to a Q Exactive mass spectrometer (Thermo Fisher). Peptides were separated on 16-cm frit-less silica emitters (New Objective, $0.75 \mu \mathrm{m}$ inner diameter) and packed in-house with reversed-phase ReproSil-Pur C18 AQ 1.9-um resin (Dr. Maisch). Peptides $(0.5 \mu \mathrm{g})$ were loaded on the column and eluted for $115 \mathrm{~min}$ using a segmented linear gradient of 5-95\% solvent B ( $80 \% \mathrm{ACN}, 0.1 \% \mathrm{FA})(0-5 \mathrm{~min}, 5 \%$; 5-65 min, $20 \%$; 65-90 min, 35\%; 90-100 $\mathrm{min}, 55 \%$; $100-115 \mathrm{~min}, 95 \%$ ) at a flow rate of $300 \mathrm{nl} \mathrm{min}^{-1}$. Mass spectra were acquired in data-dependent acquisition mode with the TOP15 method. Mass spectrometry spectra were acquired in the Orbitrap analyser with a mass range of $300-1,750 \mathrm{~m} / \mathrm{z}$ at a resolution of 70,000 full width at half maximum (FWHM) and a target value of $3 \times 10^{6}$ ions. Precursors were selected with an isolation window of $1.3 \mathrm{~m} / z$ (Q Exactive Plus) or $2.0 \mathrm{~m} / \mathrm{z}$ (Q Exactive). HCD fragmentation was performed at a normalized collision energy of 25. MS/MS spectra were acquired with a target value of $10^{5}$ ions at a resolution of 17,500 FWHM, a maximum injection time of $55 \mathrm{~ms}$ and a fixed first mass of $100 \mathrm{~m} / z$. Peptides with a charge of $+1,>6$ or with unassigned charge state were excluded from fragmentation for $\mathrm{MS}^{2}$; dynamic exclusion for $30 \mathrm{~s}$ prevented repeated selection of precursors. The mass spectrometry proteomics data are available at the ProteomeXchange Consortium via the PRIDE partner repository with the dataset identifier PXD015839.

Protein identification and quantification. Raw data were processed using MaxQuant software (v.1.5.7.4, http://www.maxquant.org//) ${ }^{45}$ to calculate label-free quantification (LFQ) and iBAQ values ${ }^{46}$. MS/MS spectra were searched for by the Andromeda search engine against a combined database containing the amino acid sequences of Pto (The Pseudomonas Genome Database) and 248 common contaminant proteins and decoy sequences. Trypsin specificity was required, and a maximum of two missed cleavages were accepted. Minimal peptide length was set to seven amino acids. Carbamidomethylation of cysteine residues was set as a fixed modification, and oxidation of methionine and protein $\mathrm{N}$-terminal acetylation as variable. Peptide-spectrum matches and proteins were retained when FDR $<1 \%$.

Data analysis. Normalized iBAQ values were used for statistical analysis because the samples analysed in the present study, and which contain bacterial and plant proteins in different ratios, do not fulfil an assumption used in LFQ analysis that the abundance of the majority of proteins does not change between conditions. For simplification and comparison to transcriptome data, information on protein groups was omitted and only the identifier displayed in the MaxQuant column Fasta header was used. For each sample, iBAQ values were normalized by TMM normalization in the package edge $\mathrm{R}^{47}$. For this, normalization factors were calculated with the calcNormFactors function, with default settings using proteins with $\mathrm{iBAQ}$ values $>0$ in all samples. When $\mathrm{BAAQ}$ values were zero in more than one replicate out of three, the protein was defined as 'not detected' and the $\mathrm{BAAQ}$ values of all replicates were converted to not available (NA). TMM-normalized iBAQ values were then $\log _{2}$-transformed. We compared TMM-normalized $\mathrm{BAAQ}$ values and $L F Q$ values using in vitro $(\mathrm{KB})$ samples, in which $L F Q$ values are not biased by the contamination of plant-derived proteins. These values showed a strong correlation $\left(R^{2}=0.94\right.$; Extended Data Fig. $\left.2 \mathrm{~b}\right)$, suggesting that TMM-normalized iBAQ values can be used as an alternative to LFQ values. After log transformation, TMM-normalized iBAQ values showed normal distribution (Extended Data Fig. 2c). To each protein, a linear model was fitted using the function $\operatorname{lmFit}$ in the limma package with the following terms: Sgptr $=$ GPTgpt $+R r+$ egtr, where $\mathrm{S}$ is the $\log _{2}$ count per million, GPT is the host genotype (or liquid medium)/Pto strain/time point interaction and the random factors, $\mathrm{R}$ is the biological replicate and $e$ is the residual. The eBayes function in the limma package was used for variance shrinkage in the calculation of $P$ values, which was then used to calculate FDR (Storey's $q$-values) using the qvalue function in the qvalue package ${ }^{39}$. To determine proteins with significant expression changes, a cut-off $q$-value $<0.01$ and $\left|\log _{2}[\mathrm{FC}]\right|>1$ were applied. Hierarchical clustering was done using either the dist and hclust functions in the Renvironment or Cluster 3.0 software ${ }^{40}$. Heat maps were created with TreeView ${ }^{41}$. Enriched GO terms were identified using BiNGO plugin for cytoscape ${ }^{42}$. Subcellular localization of bacterial proteins was obtained at www.pseudomonas.com.

GO analysis. Messenger RNA/protein expression data were separately standardized using $z$-score, and a GO expression matrix was generated by taking the mean $z$-score for each GO term. To select GO terms showing distinct expression patterns among different conditions, we performed statistical tests in all pairwise comparisons among 15 conditions for each GO term and manually curated GO terms with high numbers of significant pairs (redundant GO terms were avoided).

RT-qPCR analysis. RT-qPCR was performed using the SuperScript One-Step RT-PCR system kit (Invitrogen). As inputs, 30 ng of DNase-treated RNA extracted from infected leaves was used for analysis of bacterial genes.

Total protein extraction and immunoblotting analysis. Pto wild-type and Pto AvrRpt2 strains were infiltrated into 4-week-old A. thaliana leaves and harvested at the indicated time points. The infiltrated leaves were ground into a fine powder in liquid nitrogen. Total proteins were extracted using the phenol extraction method. Briefly, equal amounts of protein extraction buffer $(50 \mathrm{mM}$ Tris-Cl pH 7.6, $5 \mathrm{mM}$ EDTA, $5 \mathrm{mM}$ EGTA, $2 \mathrm{mM}$ DTT and protease inhibition cocktail) and water-saturated phenol were added to ground tissues and mixed well. The phenolic layer was moved to a new tube after centrifugation at $6,000 \mathrm{~g}$ for $5 \mathrm{~min}$. Total proteins were precipitated at $-20^{\circ} \mathrm{C}$ after the addition of an additional 2.5 volumes of ice-cold methanol containing $0.1 \mathrm{M}$ ammonium acetate. Precipitated proteins were then washed twice in ice-cold methanol containing $0.1 \mathrm{M}$ ammonium acetate and ice-cold $80 \% \mathrm{v} / \mathrm{v}$ acetone, respectively. Protein pellets were dissolved in protein sample buffer $(40 \% \mathrm{v} / \mathrm{v}$ glycerol, $250 \mathrm{mM}$ Tris-Cl pH 6.8, 3.5 M SDS, $5 \% \mathrm{v} / \mathrm{v} \beta$-mercaptoethanol, $0.04 \% \mathrm{w} / \mathrm{v}$ bromophenol blue) after heating at $95^{\circ} \mathrm{C}$ for $5 \mathrm{~min}$ and cooling on ice for $1 \mathrm{~min}$. For immunoblotting, proteins were separated on SDS-polyacrylamide gel electrophoresis and then transferred onto a polyvinylidene difluoride membrane using an electrophoretic apparatus. Protein detection was performed using anti- $\operatorname{HrCC}(1: 5,000)^{48}$ and anti-HrpZ $(1: 5,000)^{49}$ as primary antibodies, and anti-mouse $(1: 10,000)$ and anti-rabbit $(1: 10,000)$ conjugated with horseradish peroxidase as secondary antibodies. To compare the expression of $\mathrm{HrpZ}$ between samples, protein loading was first normalized to HrcC expression.

Primers. A list of primers used in this study is provided in Supplementary Data 12.

Reporting Summary. Further information on research design is available in the Nature Research Reporting Summary linked to this article.

\section{Data availability}

Source Data for Fig. 6b are provided with the paper. The RNA-seq data used in this study are deposited in the National Center for Biotechnology Information Gene Expression Omnibus database (accession no. GSE138901). The mass spectrometry proteomics data are available at the ProteomeXchange Consortium via the PRIDE partner repository with the dataset identifier PXD015839.

Received: 10 December 2019; Accepted: 8 May 2020; Published online: 15 June 2020

\section{References}

1. Nobori, T., Mine, A. \& Tsuda, K. Molecular networks in plant-pathogen holobiont. FEBS Lett. 592, 1937-1953 (2018).

2. Nobori, T. \& Tsuda, K. The plant immune system in heterogeneous environments. Curr. Opin. Plant Biol. 50, 58-66 (2019).

3. Nobori, T. et al. Transcriptome landscape of a bacterial pathogen under plant immunity. Proc. Natl Acad. Sci. USA 115, E3055-E3064 (2018).

4. Kunkel, B. N., Bent, A. F., Dahlbeck, D., Innes, R. W. \& Staskawicz, B. J. RPS2, an Arabidopsis disease resistance locus specifying recognition of Pseudomonas syringae strains expressing the avirulence gene avrRpt2. Plant Cell 5, 865-875 (1993).

5. Yu, G.-L., Katagiri, F. \& Ausubel, F. M. Arabidopsis mutations at the RPS2 locus result in loss of resistance to Pseudomonas syringae strains expressing the avirulence gene avrRpt. Mol. Plant Microbe Interact. 6 , 434-443 (1993).

6. Tsuda, K., Sato, M., Stoddard, T., Glazebrook, J. \& Katagiri, F. Network properties of robust immunity in plants. PLOS Genet. 5, e1000772 (2009).

7. Zhang, W. et al. Plant-necrotroph co-transcriptome networks illuminate a metabolic battlefield. eLife 8, 1-32 (2019).

8. O'Connell, R. J. et al. Lifestyle transitions in plant pathogenic Colletotrichum fungi deciphered by genome and transcriptome analyses. Nat. Genet. 44, 1060-1065 (2012)

9. Abreu, R., de, S., Penalva, L. O., Marcotte, E. M. \& Vogel, C. Global signatures of protein and mRNA expression levels. Mol. Biosyst. 5, 1512-1526 (2009).

10. Maier, T., Güell, M. \& Serrano, L. Correlation of mRNA and protein in complex biological samples. FEBS Lett. 583, 3966-3973 (2009).

11. Müller, D. B., Schubert, O. T., Röst, H., Aebersold, R. \& Vorholt, J. A. Systems-level proteomics of two ubiquitous leaf commensals reveals complementary adaptive traits for phyllosphere colonization. Mol. Cell. Proteomics 15, 3256-3269 (2016). 
12. Levy, A., Conway, J. M., Dangl, J. L. \& Woyke, T. Elucidating bacterial gene functions in the plant microbiome. Cell Host Microbe 24, 475-485 (2018).

13. Llamas, A., Imperi, F., Visca, P. \& Lamont, I. L. Cell-surface signaling in Pseudomonas: stress responses, iron transport, and pathogenicity. FEMS Microbiol. Rev. 38, 569-597 (2014)

14. Lu, P., Vogel, C., Wang, R., Yao, X. \& Marcotte, E. M. Absolute protein expression profiling estimates the relative contributions of transcriptional and translational regulation. Nat. Biotechnol. 25, 117-124 (2007).

15. Ishihama, Y. et al. Index (emPAI) for estimation of absolute protein amount in proteomics by the number of sequenced peptides per protein. Mol. Cell. Proteomics 4, 1265-1272 (2005).

16. Corbin, R. W. et al. Toward a protein profile of Escherichia coli: comparison to its transcription profile. Proc. Natl Acad. Sci. USA 100, 9232-9237 (2003).

17. Toruño, T. Y., Stergiopoulos, I. \& Coaker, G. Plant-pathogen effectors: cellular probes interfering with plant defenses in spatial and temporal manners. Annu. Rev. Phytopathol. 54, 419-441 (2016).

18. Sreedharan, A., Penaloza-Vazquez, A., Kunkel, B. N. \& Bender, C. L. CorR regulates multiple components of virulence in Pseudomonas syringae $\mathrm{pv}$. tomato DC3000. Mol. Plant. Microbe. Interact. 19, 768-779 (2006).

19. Gygi, S. P., Rochon, Y., Franza, B. R. \& Aebersold, R. Correlation between protein and mRNA abundance in yeast. Mol. Cell. Biol. 19, 1720-1730 (1999).

20. Ghazalpour, A. et al. Comparative analysis of proteome and transcriptome variation in mouse. PLoS Genet. 7, e1001393 (2011).

21. Foss, E. J. et al. Genetic basis of proteome variation in yeast. Nat. Genet. 39, 1369-1375 (2007).

22. Lan, P., Li, W. \& Schmidt, W. Complementary proteome and transcriptome profiling in phosphate-deficient Arabidopsis roots reveals multiple levels of gene egulation. Mol. Cell. Proteomics 11, 1156-1166 (2012).

23. Marguerat, S. et al. Quantitative analysis of fission yeast transcriptomes and proteomes in proliferating and quiescent cells. Cell 151, 671-683 (2012).

24. Yao, J. \& Allen, C. Chemotaxis is required for virulence and competitive fitness of the bacterial wilt pathogen Ralstonia solanacearum. J. Bacteriol. 188, 3697-3708 (2006).

25. Papenfort, K. \& Bassler, B. L. Quorum sensing signal-response systems in Gram-negative bacteria. Nat. Rev. Microbiol. 14, 576-588 (2016).

26. Kvitko, B. H., Ramos, A. R., Morello, J. E., Oh, H. S. \& Collmer, A. Identification of harpins in Pseudomonas syringae pv. tomato DC3000, which are functionally similar to HrpK1 in promoting translocation of type III secretion system effectors. J. Bacteriol. 189, 8059-8072 (2007).

27. Petnicki-Ocwieja, T., Van Dijk, K. \& Alfano, J. R. The hrpK operon of Pseudomonas syringae pv. tomato DC3000 encodes two proteins secreted by the type III (Hrp) protein secretion system: HopB1 and HrpK, a putative type III translocator. Society 187, 649-663 (2005).

28. Wang, Y. et al. Site-specific cleavage of bacterial MucD by secreted proteases mediates antibacterial resistance in Arabidopsis. Nat. Commun. 10, 2853 (2019).

29. Mackey, D., Belkhadir, Y., Alonso, J. M., Ecker, J. R. \& Dangl, J. L. Arabidopsis RIN4 is a target of the type III virulence effector AvrRpt2 and modulates RPS2-mediated resistance. Cell 112, 379-389 (2003).

30. Jirage, D. et al. Arabidopsis thaliana PAD4 encodes a lipase-like gene that is important for salicylic acid signaling. Proc. Natl Acad. Sci. USA 96, 13583-13588 (1999).

31. Wildermuth, M. C., Dewdney, J., Wu, G. \& Ausubel, F. M. Isochorismate synthase is required to synthesize salicylic acid for plant defence. Nature $\mathbf{4 1 4}$ 562-565 (2001).

32. Tsuda, K. et al. Dual regulation of gene expression mediated by extended MAPK activation and salicylic acid contributes to robust innate immunity in Arabidopsis thaliana. PLoS Genet. 9, e1004015 (2013).

33. Kvitko, B. H. \& Collmer, A. Construction of Pseudomonas syringae pv. tomato DC3000 mutant and polymutant strains. Methods Mol. Biol. 712, 109-128 (2011).

34. Huynh, T. V., Dahlbeck, D. \& Staskawicz, B. J. Bacterial blight of soybean: regulation of a pathogen gene determining host cultivar specificity. Science 245, 1374-1377 (1989).

35. Tsuda, K., Sato, M., Glazebrook, J., Cohen, J. D. \& Katagiri, F. Interplay between MAMP-triggered and SA-mediated defense responses. Plant J. 53, 763-775 (2008).

36. Nobori, T. \& Tsuda, K. In planta transcriptome analysis of Pseudomonas syringae. Bio Protoc. 8, 1-8 (2018).
37. Langmead, B. \& Salzberg, S. L. Fast gapped-read alignment with Bowtie 2. Nat. Methods 9, 357-360 (2012).

38. Anders, S., Pyl, P. T. \& Huber, W. HTSeq A Python framework to work with high-throughput sequencing data. Bioinformatics 31, 166-169 (2014).

39. Storey, J. D. \& Tibshirani, R. Statistical significance for genomewide studies. Proc. Natl Acad. Sci. USA 100, 9440-9445 (2003).

40. Hoon, M. J. L. De, Imoto, S., Nolan, J. \& Miyano, S. Open source clustering software. Bioinformatics 20, 1453-1454 (2004).

41. Eisen, M. B., Spellman, P. T., Brown, P. O. \& Botstein, D. Cluster analysis and display of genome-wide expression patterns. Proc. Natl Acad. Sci. USA 95, 12930-12933 (1998)

42. Maere, S., Heymans, K. \& Kuiper, M. Systems biology BiNGO: a Cytoscape plugin to assess overrepresentation of Gene Ontology categories in Biological Networks. Bioinformatics 21, 3448-3449 (2005).

43. Bronstein, P. A. et al. Global transcriptional responses of Pseudomonas syringae DC3000 to changes in iron bioavailability in vitro. BMC Microbiol. 8, 209 (2008).

44. Borner, G. H. H. \& Fielding, A. B. Using in-solution digestion, peptide fractionation, and a qexactive mass spectrometer to analyze the proteome of clathrin-coated vesicles. Cold Spring Harb. Protoc. 2014, 1192-1195 (2014).

45. Cox, J. \& Mann, M. MaxQuant enables high peptide identification rates, individualized p.p.b.-range mass accuracies and proteome-wide protein quantification. Nat. Biotechnol. 26, 1367-1372 (2008).

46. Tyanova, S., Temu, T. \& Cox, J. The MaxQuant computational platform for mass spectrometry-based shotgun proteomics. Nat. Protoc. 11, 2301-2319 (2016).

47. Robinson, M. D., McCarthy, D. J. \& Smyth, G. K. edgeR: a Bioconductor package for differential expression analysis of digital gene expression data. Bioinformatics 26, 139-140 (2010).

48. Lin, Y., Hsu, S. \& Huang, H. Evidence for the interaction between HrpT and HrcC of Pseudomonas syringae pv. syringae 61. Plant Pathol. Bull. 15, 171-185 (2006)

49. Alfano, J. R., Bauer, D. W., Milos, T. M. \& Collmer, A. Analysis of the role of the Pseudomonas syringae pv. syringae HrpZ harpin in elicitation of the hypersensitive response in tobacco using functionally non-polar hrpZ deletion mutations, truncated HrpZ fragments, and hrmA mutations. Mol. Microbiol. 19, 715-728 (1996).

\section{Acknowledgements}

We thank S. Yang He for providing the HrpZ antibody, H.-C. Huang for providing the $\mathrm{HrcC}$ antibody and A. Harzen and K. Kramer for support in sample preparation and mass spectrometry analysis. We thank A. Collmer for the pCPP5040 plasmid, the Max Planck Genome Centre for sequencing support and N. Donnelly for critical comments on the manuscript. This work was supported by the Huazhong Agricultural University Scientific \& Technological Self-innovation Foundation, the Max Planck Society, a German Research Foundation grant (no. SPP2125 to K.T.), a predoctoral fellowship from the Nakajima Foundation (to T.N.) and a postdoctoral fellowship from the Alexander von Humboldt Foundation (to Y.W.)

\section{Author contributions}

T.N. and K.T. designed the research. T.N., Y.W., J.W., S.C.S., Y.T., I.F. and H.N. performed experiments. T.N. and K.T. wrote the paper with input from all authors.

\section{Competing interests}

The authors declare no competing interests.

\section{Additional information}

Extended data is available for this paper at https://doi.org/10.1038/s41477-020-0690-7.

Supplementary information is available for this paper at https://doi.org/10.1038/ s41477-020-0690-7.

Correspondence and requests for materials should be addressed to K.T.

Peer review information Nature Plants thanks Asaf Levy and the other, anonymous, reviewer(s) for their contribution to the peer review of this work.

Reprints and permissions information is available at www.nature.com/reprints.

Publisher's note Springer Nature remains neutral with regard to jurisdictional claims in published maps and institutional affiliations.

(c) The Author(s), under exclusive licence to Springer Nature Limited 2020 
a
RNA-seq
\begin{tabular}{|l|} 
RE $\left(\log _{2}\right)$ \\
\hline \\
\hline-3
\end{tabular}

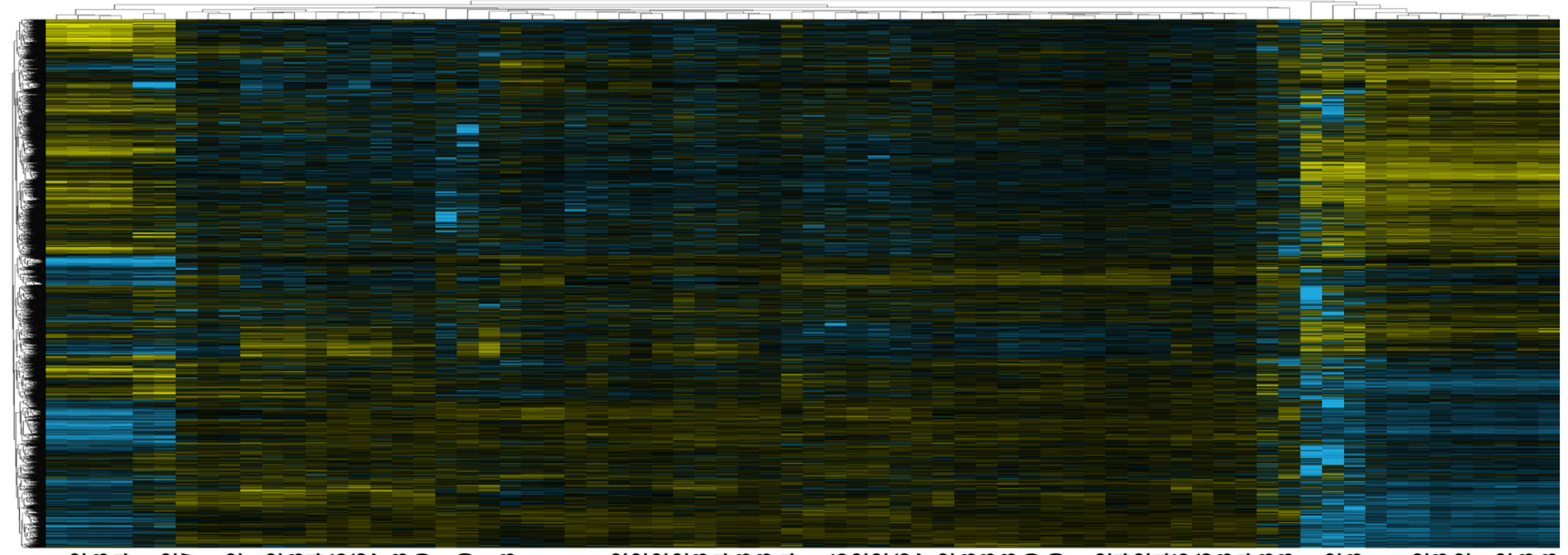

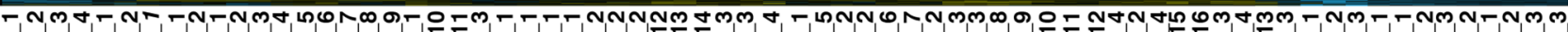

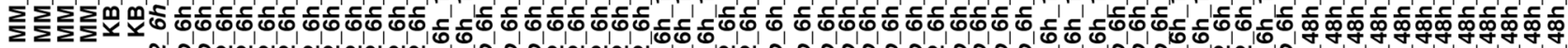

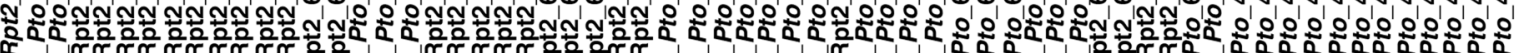
急

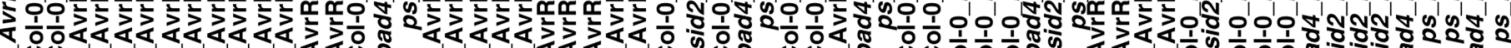
10000

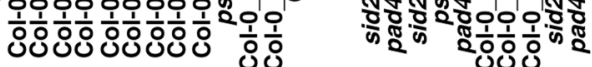
ठํㅇㅇ

b
Proteome
RE $\left(\log _{2}\right)$
$-3 \quad 3$

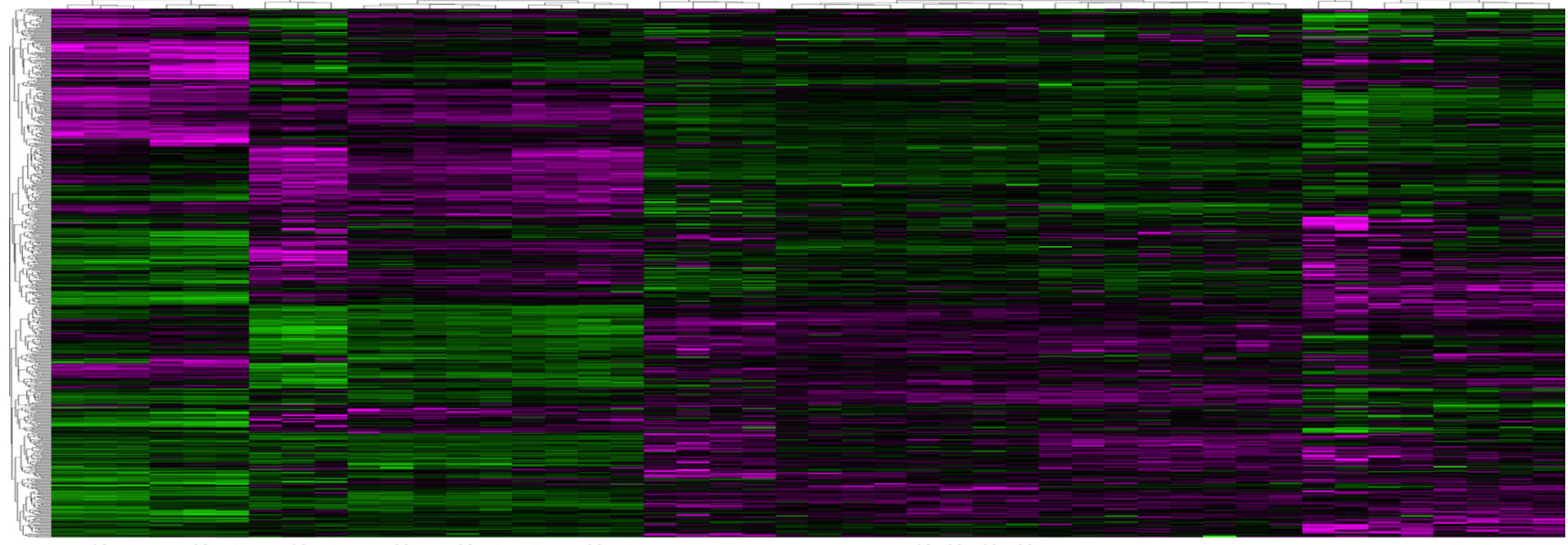

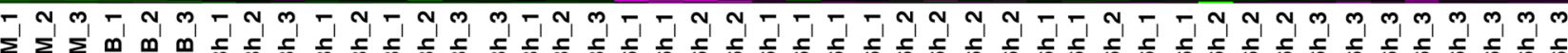

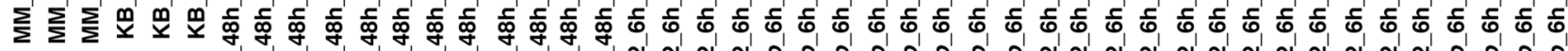

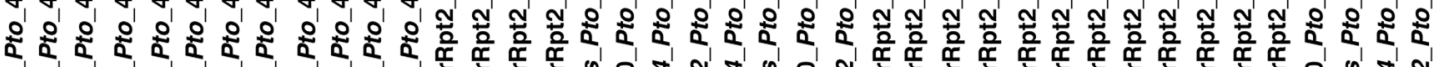

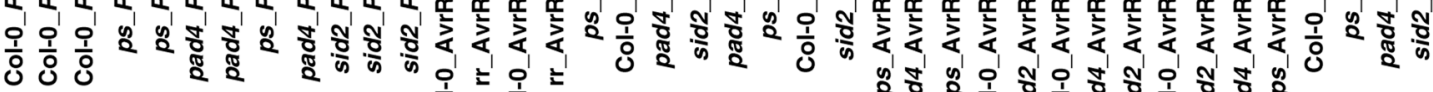

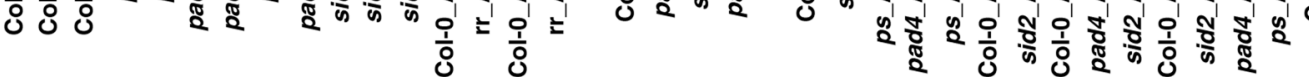

Extended Data Fig. 1 | Overview of RNA-seq and proteome datasets. Hierarchical clustering of relative expression (RE) of (a) RNA-seq data and (b) proteome data that were normalized separately. a, b, ps, pad4 sid2; rr, rpm1 rps2. 


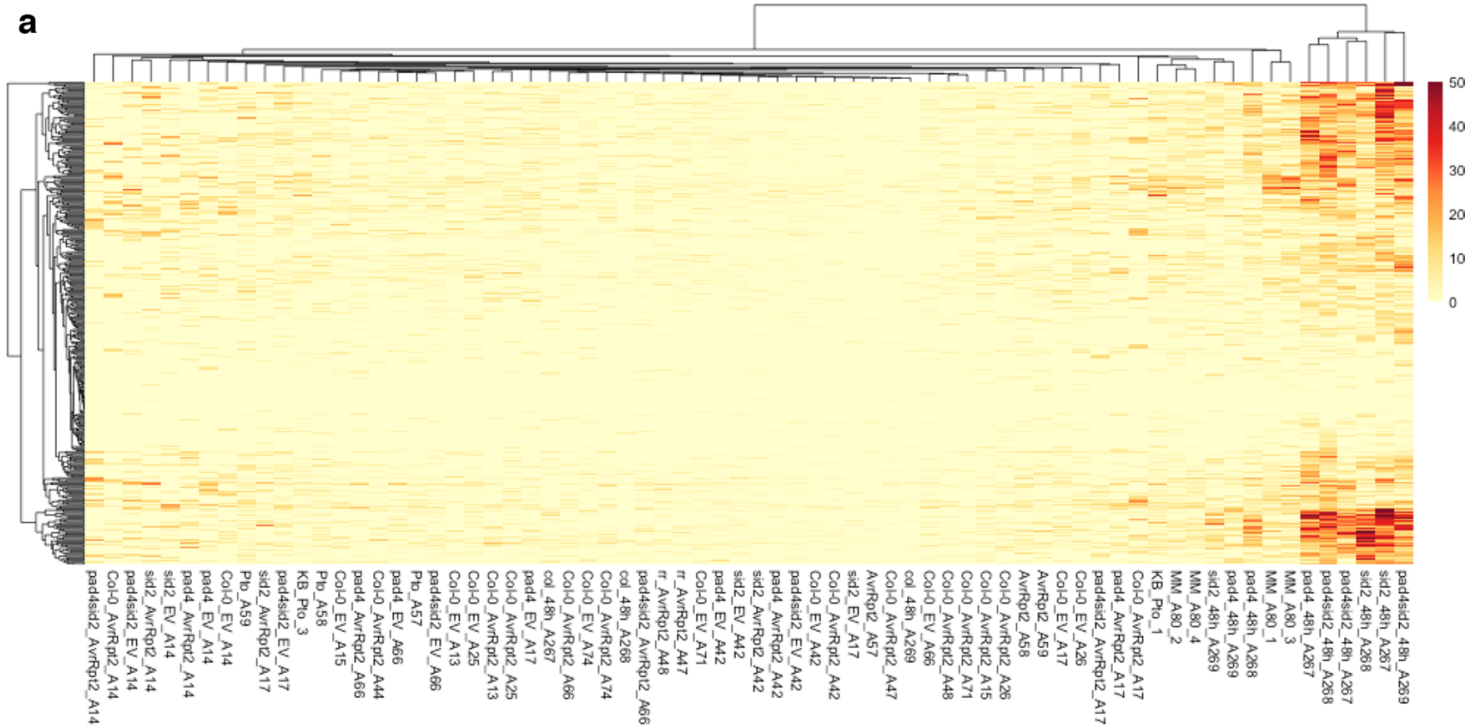

b

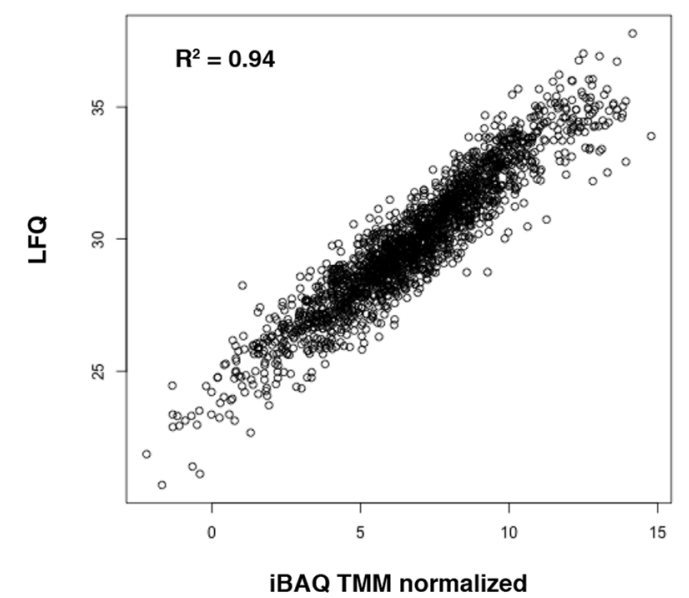

C

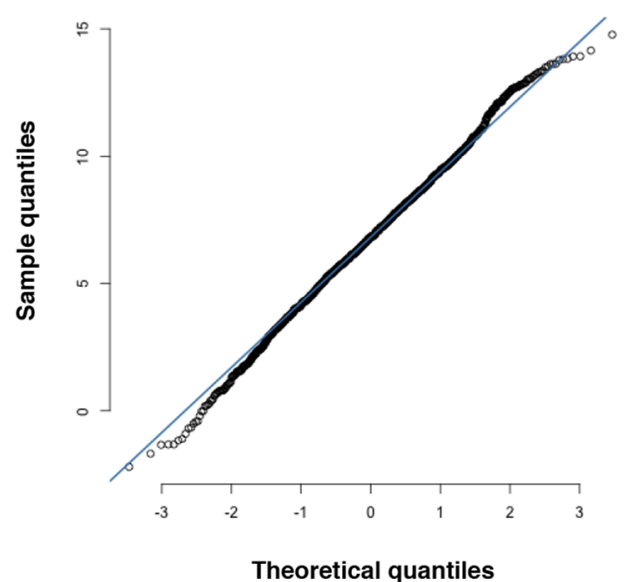

Extended Data Fig. 2 | Normalization of RNA-seq and proteome data. a, Heatmap of RNA-seq count data displaying 387 genes that are omitted from further analyses due to low average count $(<5)$. Genes with average counts of zero were not displayed. $\mathbf{b}$, Scatter plot comparing TMM-normalized iBAQ values and LFQ values of proteome data. The Pearson's correlation coefficient was shown. (C) Q-Q plot of TMM-normalized iBAQ values. b, c, In vitro (KB) samples were used $(n=3)$. 


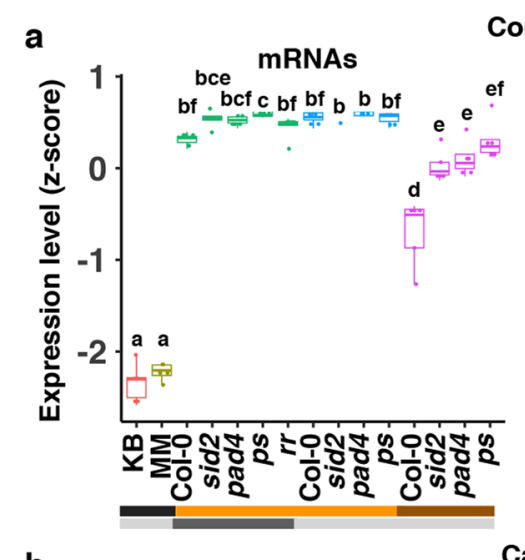

Coronatine

b
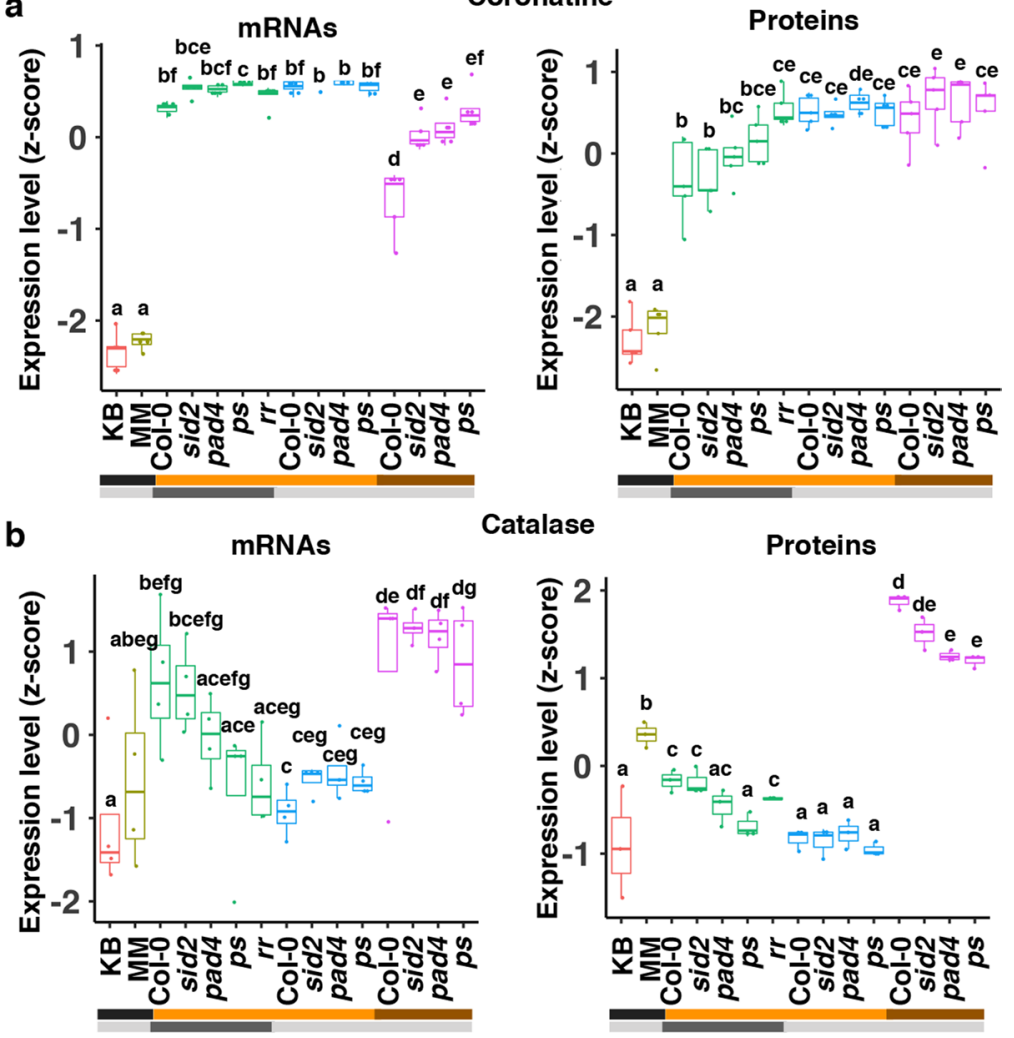

Catalase Proteins

In vitro

In planta 6 hpi

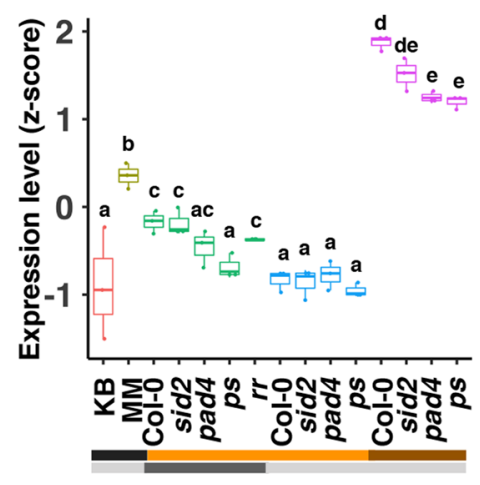

In planta 48 hpi

Pto AvrRpt2

c

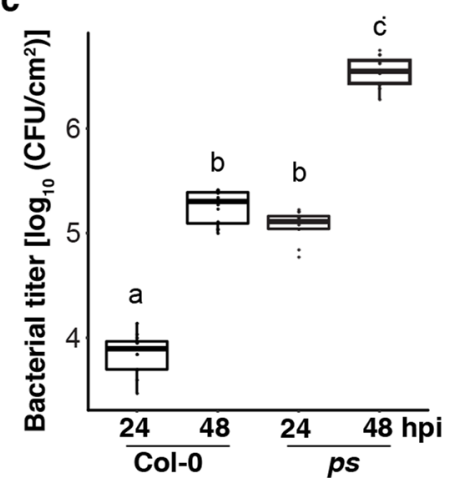

Extended Data Fig. 3 | Regulation of selected mRNAs and proteins. a, b, Expression (z-score) of mRNAs (left) and proteins (right) related to "coronatine biosynthesis" and "catalase" under various conditions. Light and dark gray sidebars represent Pto and Pto AvrRpt2, respectively. Black, orange, and brown sidebars represent in vitro (KB), in planta 6 hpi, and in planta 48 hpi, respectively. MM, minimal medium; ps, pad4 sid2; rr, rpm1 rps2. See Supplementary Data 13 for the sample size. Different letters indicate statistically significant differences (adjusted $p$-value $<0.01$; two-tailed Student's $t$ test followed by Benjamini-Hochberg method). c, Growth of Pto (infiltrated at $\mathrm{OD}_{600}=0.005$ ) in Col-0 and pad 4 sid 2 at 24 and 48 hpi. $\mathrm{n}=12$ biological replicates from three independent experiments. Different letters indicate statistically significant differences (adjusted $p$-value $<0.01$; two-tailed Student's $t$ test followed by Benjamini-Hochberg method). a-c, Results are shown as box plots with boxes displaying the 25th-75th percentiles, the centerline indicating the median, whiskers extending to the minimum and maximum values no further than 1.5 inter-quartile range. 

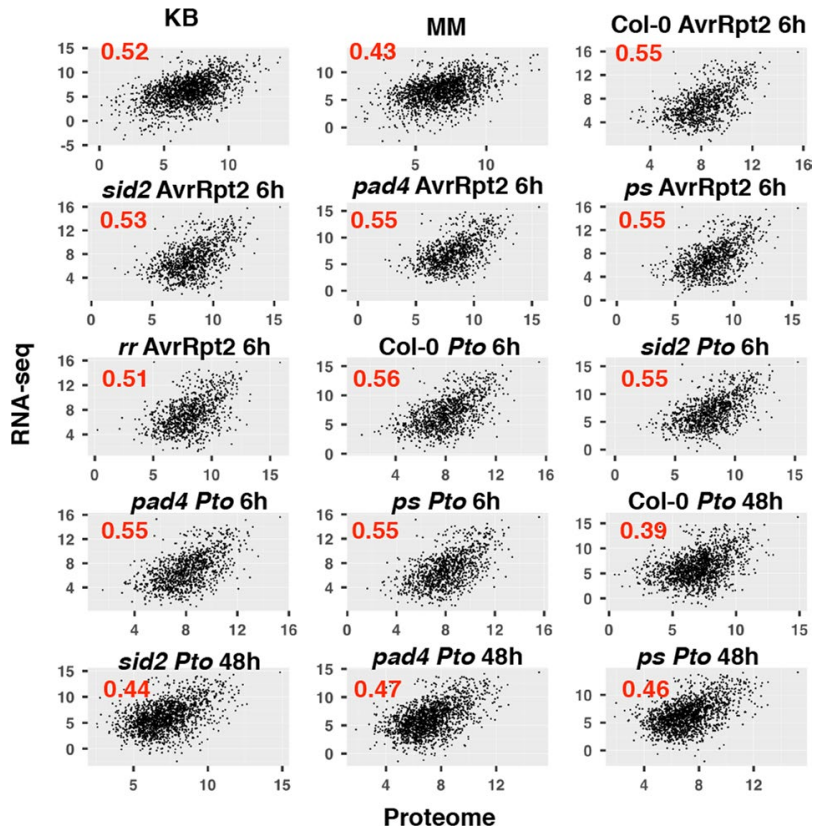

Extended Data Fig. 4 | Integration of bacterial transcriptome and proteome data. Comparisons between transcriptome and proteome data in each condition. The Pearson's correlation coefficients were shown. mRNAs/proteins detected in both the transcriptome and proteome in each condition were used for this analysis. See Supplementary Data 13 for the sample size. 


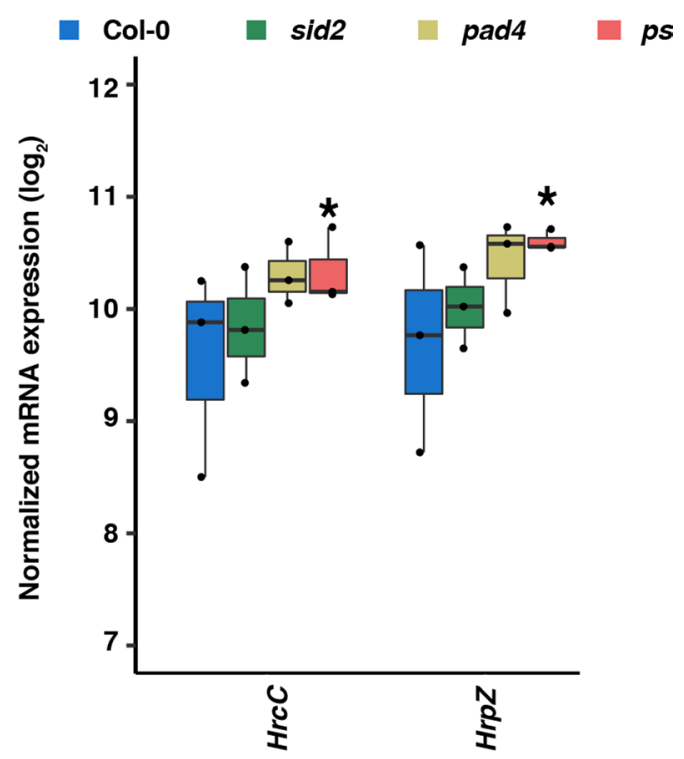

Extended Data Fig. 5 | mRNA expression of genes comprising T3SS based on RNA-seq. Expression of hrcC and hrpZ after TMM normalization was shown. Asterisks indicate statistically significant differences in mutants relative to Col-0 after fitting linear model (adjusted P $<0.01$; two-tailed Student's $t$ test followed by Benjamini-Hochberg method). ps, pad4 sid2. See Supplementary Data 13 for the sample size. Results are shown as box plots with boxes displaying the 25th-75th percentiles, the centerline indicating the median, whiskers extending to the minimum and maximum values no further than 1.5 inter-quartile range. 


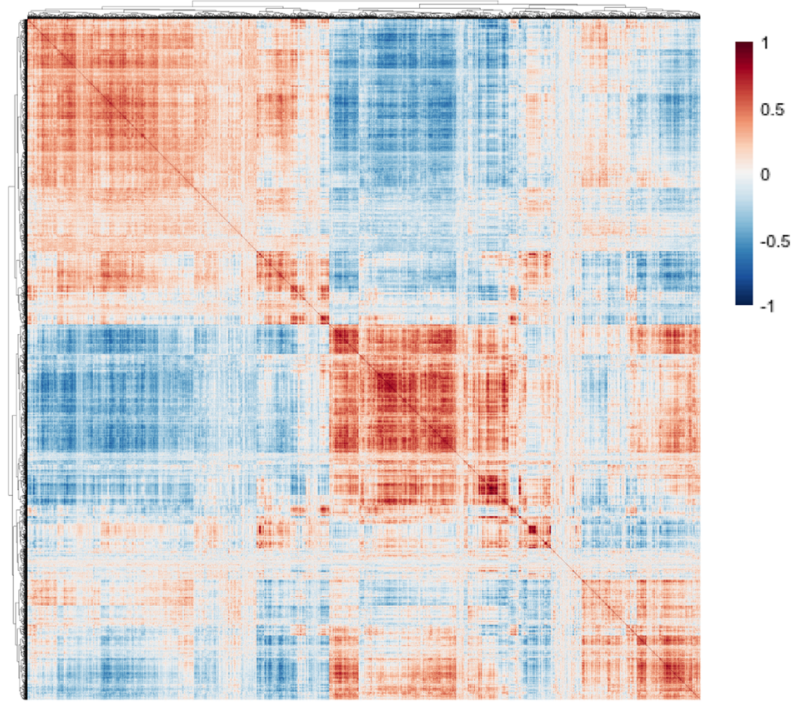

b

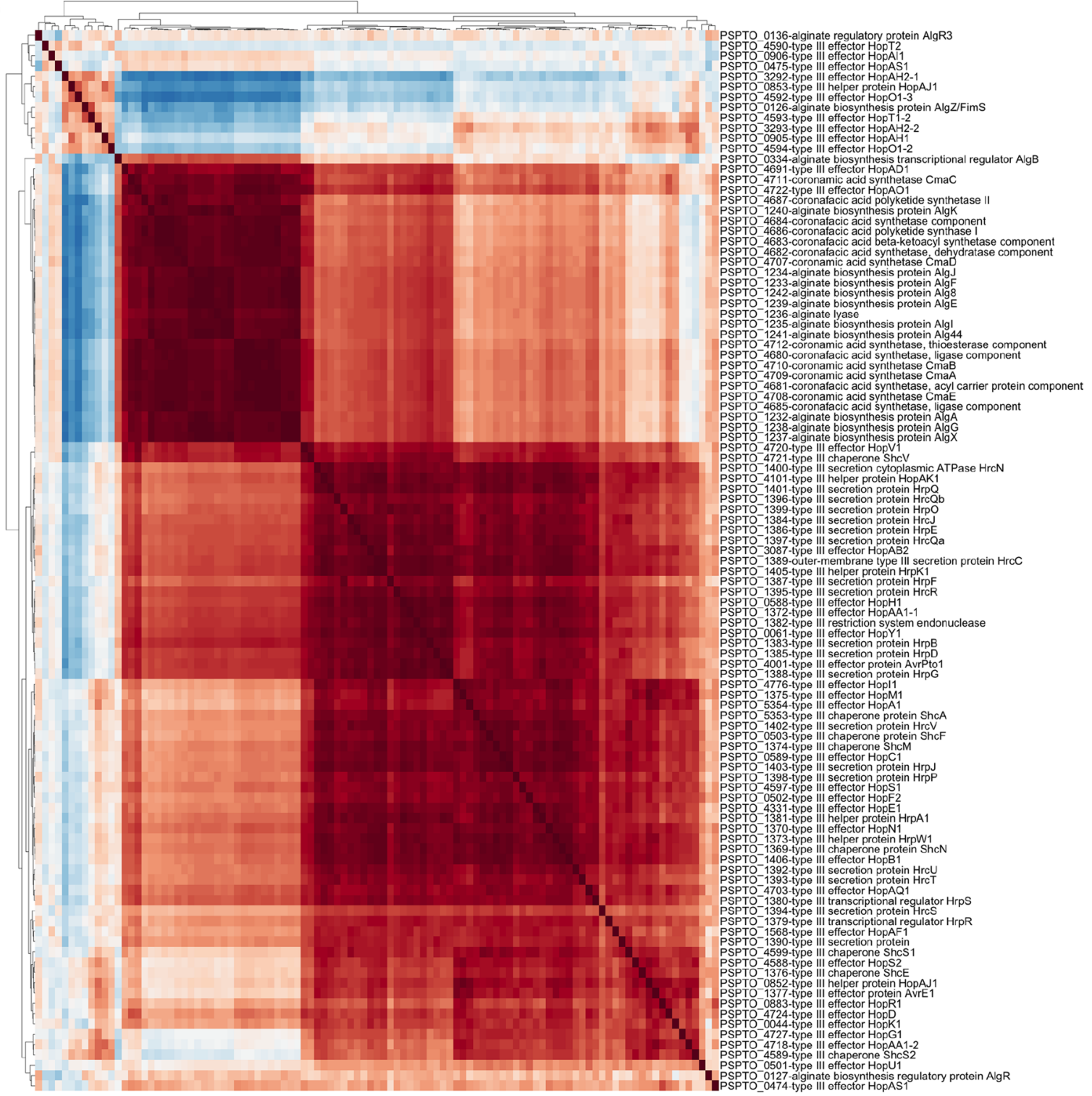

Extended Data Fig. 6 | Co-expression analysis of Pto mRNAs. a, Correlation matrix of 4,765 Pto mRNAs based on 125 transcriptome datasets in 38 conditions. $\mathbf{b}$, Correlation matrix of Pto mRNAs related to coronatine, alginate, and the type III secretion system (T3SS). $\mathbf{a}$, b, The Pearson's correlation coefficients were used. 


\section{Reporting Summary}

Nature Research wishes to improve the reproducibility of the work that we publish. This form provides structure for consistency and transparency in reporting. For further information on Nature Research policies, see Authors \& Referees and the Editorial Policy Checklist.

\section{Statistics}

For all statistical analyses, confirm that the following items are present in the figure legend, table legend, main text, or Methods section.

$\mathrm{n} / \mathrm{a}$ | Confirmed

$\square$ \The exact sample size $(n)$ for each experimental group/condition, given as a discrete number and unit of measurement

$\square$ \ A statement on whether measurements were taken from distinct samples or whether the same sample was measured repeatedly

The statistical test(s) used AND whether they are one- or two-sided

$\square$ Only common tests should be described solely by name; describe more complex techniques in the Methods section.

$\bigotimes \square$ A description of all covariates tested

$\square$ \ A description of any assumptions or corrections, such as tests of normality and adjustment for multiple comparisons

$\square$ A full description of the statistical parameters including central tendency (e.g. means) or other basic estimates (e.g. regression coefficient)

AND variation (e.g. standard deviation) or associated estimates of uncertainty (e.g. confidence intervals)

$\square$ For null hypothesis testing, the test statistic (e.g. $F, t, r$ ) with confidence intervals, effect sizes, degrees of freedom and $P$ value noted

$\square$ Give P values as exact values whenever suitable.

Х $\square$ For Bayesian analysis, information on the choice of priors and Markov chain Monte Carlo settings

$\square \bigotimes$ For hierarchical and complex designs, identification of the appropriate level for tests and full reporting of outcomes

$\square$ Estimates of effect sizes (e.g. Cohen's $d$, Pearson's $r$ ), indicating how they were calculated

Our web collection on statistics for biologists contains articles on many of the points above.

\section{Software and code}

Policy information about availability of computer code

Data collection Hybrid Quadrupole-Orbitrap Mass Spectrometer (Thermo), MaxQuant (v1.5.7.4), Illumina HiSeq 3000

Data analysis MaxQuant (v1.5.7.4), Bowtie2 (v2.1.1), HTSeq (v0.6.1), samtools (v1.3.1), R (v3.6.0), RStudio (v1.1.453), cytoscape (v3.4.0), Cluster3.0 TreeView(v1.1.6r4)

For manuscripts utilizing custom algorithms or software that are central to the research but not yet described in published literature, software must be made available to editors/reviewers. We strongly encourage code deposition in a community repository (e.g. GitHub). See the Nature Research guidelines for submitting code \& software for further information.

\section{Data}

Policy information about availability of data

All manuscripts must include a data availability statement. This statement should provide the following information, where applicable:

- Accession codes, unique identifiers, or web links for publicly available datasets

- A list of figures that have associated raw data

- A description of any restrictions on data availability

The RNA sequencing data used in this study are deposited in the National Center for Biotechnology Information Gene Expression Omnibus database (accession no. GSE138901). The mass spectrometry proteomics data are available at the ProteomeXchange Consortium via the PRIDE partner repository with the data set identifier PXD015839. 


\section{Field-specific reporting}

Please select the one below that is the best fit for your research. If you are not sure, read the appropriate sections before making your selection. $\bigotimes$ Life sciences $\quad \square$ Behavioural \& social sciences $\quad \square$ Ecological, evolutionary \& environmental sciences

For a reference copy of the document with all sections, see nature.com/documents/nr-reporting-summary-flat.pdf

\section{Life sciences study design}

All studies must disclose on these points even when the disclosure is negative.

Sample size For RNA-seq and proteome, approximately 100 leaves (four leaves per plant) were collected for each sample. RNA-seq and proteome data contain at least three biological replicates from independent experiments. In our previous study (Nobori et al., 2018, PNAS), RNA-seq data were highly reproducible and statistical analyses with three replicates yielded biologically relevant results. Therefore, we prepared three replicates in this study; and the data were highly reproducible (see Extended Data Fig1).

Data exclusions No data were excluded for analysis.

Replication For each experiment, at least three replicates were made. All attempts at replication were successful.

Randomization For each experiment, different plant genotypes were grown in the same condition and were placed randomly in the growth room.

Blinding Not applicable since no group allocation was conducted in this study.

\section{Reporting for specific materials, systems and methods}

We require information from authors about some types of materials, experimental systems and methods used in many studies. Here, indicate whether each material, system or method listed is relevant to your study. If you are not sure if a list item applies to your research, read the appropriate section before selecting a response.

Materials \& experimental systems

\begin{tabular}{c|l}
\hline $\mathrm{n} / \mathrm{a}$ & Involved in the study
\end{tabular}

$\square$ Antibodies

$\bigotimes \square$ Eukaryotic cell lines

\ $\square$ Palaeontology

Methods

n/a Involved in the study

\ $\square$ chlp-seq

Х $\square$ Flow cytometry

$\square$ Animals and other organisms

\ $\square$ Human research participants

Х $\square$ Clinical data

\section{Antibodies}

Antibodies used

HrpZ and HrcC antibodies were provided by Dr. Sheng Yang He and Dr. Hsiou-Chen Huang, respectively.

Validation

The HrcC antibody was validated in Lin et al., 2006 Plant Pathology Bulletin. The HrpZ antibody was validated in He et al., 1993, Cell.

\section{Animals and other organisms}

Policy information about studies involving animals; ARRIVE guidelines recommended for reporting animal research

Laboratory animals

Wild animals

Field-collected samples

Ethics oversight
This study did not involve laboratory animals.

This study did not involve wild animals.

This study did not involve field-collected samples.

No ethical approval was required.

Note that full information on the approval of the study protocol must also be provided in the manuscript. 